\title{
Analysis of a finite volume-finite element method for Darcy-Brinkman two-phase flows in porous media
}

\author{
Houssein NASSER EL DINE(1,2), Mazen SAAD ${ }^{(1)}$
}

\begin{abstract}
In this paper, we are interested in the displacement of two incompressible phases in a Darcy-Brinkman flow in a porous media. The equations are obtained by the conservation of the mass and by considering the Brinkman regularization velocity of each phase. This model is treated in its general form with the whole nonlinear terms. This paper deals with construction and convergence analysis of a combined finite volume- non conforming finite element scheme together with a phase-by-phase upstream according to the total velocity.
\end{abstract}

\section{The Darcy-Brinkman model}

Different empiric laws are used to describe the filtration of a fluid through porous media. Darcy law is the most popular one, due to its simplicity. It states that the filtration velocity of the fluid is proportional to the pressure gradient. Darcy law cannot sustain the no-slip condition on an impermeable wall or a transmission condition on the contact with free flow $[18,22]$. That motivated H. Brinkmann in 1947 to modify the Darcy law in order to be able to impose the no-slip boundary condition on an obstacle submerged in porous medium $[7,17,18]$. He assumed large permeability to compare his law with experimental data and assumed that the second viscosity $\mu$ equals to the physical viscosity of the fluid in the case of monophasic flow. Brinkman law could formally be obtained from the Stokes system describing the microscopic flow, by adding the resistance to the flow [2].

Let $T>0$, fixed and let $\Omega$ be a bounded set of $\mathbb{R}^{d}(d \geq 1)$. We set $Q_{T}=(0, T) \times \Omega, \Sigma_{T}=(0, T) \times \partial \Omega$, and $\eta$ is the outward normal to the boundary $\partial \Omega$. The mass conservation of two incompressible phases with DarcyBrinkman velocity of each phase can be written in the following system, (see $[11,16]$ for more details),

$$
\begin{cases}\phi \partial_{t} s-\mu \phi \Delta \partial_{t} s-\operatorname{div}(f(s) \lambda(s) \Lambda \nabla p)-\operatorname{div}(\alpha(s) \Lambda \nabla s)=0, & \text { in } Q_{T} \\ -\operatorname{div}(\lambda(s) \Lambda \nabla p)=0, & \text { in } Q_{T} \\ \int_{\Omega} p(t, x) \mathrm{d} x=0, & \text { in }(0, T) \\ \lambda(s) \Lambda \nabla p(t, x) \cdot \eta=\pi(t, x), & \text { on } \Sigma_{T} \\ \mu \phi \nabla \partial_{t} s \cdot \eta+f(s) \pi+\alpha(s) \Lambda \nabla s \cdot \eta=h(t, x), & \text { on } \Sigma_{T} \\ s(0, x)=s_{0}(x), & \text { in } \Omega .\end{cases}
$$

In the above model, the first saturation phase (volume fraction) is represented by $s=s(t, x)$ and the other saturation phase is stated to be $1-s$ and by $p=p(t, x)$ the global pressure. Next, $\lambda(s)=\frac{\lambda_{1}(s)}{\mu_{1}}+\frac{\lambda_{2}(s)}{\mu_{2}}$ is the total mobility with $\lambda_{i}$ and $\mu_{i}$ are the relative permeability and the viscosity of the phase $i=1,2$; where $\Lambda$ is the permeability tensor of the porous medium and $f(s)=\frac{\lambda_{1}(s)}{\mu_{1} \lambda(s)}$ is the fractional flow. Furthermore, the function

(1)- École Centrale de Nantes. UMR 6629 CNRS, Laboratoire de Mathmatiques Jean Leray. F-44321, Nantes, France

(2)- Lebanese University, Doctoral School of Sciences and Technology (EDST), Laboratory of Mathematics, Hadath-lebanon 
$\alpha(s)=\frac{\lambda_{1}(s) \lambda_{2}(s)}{\mu_{1} \mu_{2} \lambda(s)} p_{c}^{\prime}(s)$ is the capillary pressure term. The viscosity $\mu>0$ is said the Brinkman viscosity.

We give the main assumptions made about the system:

(H1) $\Lambda$ is a symmetric matrix where $\Lambda_{i j} \in L^{\infty}(\Omega)$, and there exists a constant $C_{\Lambda}>0$ such that $\Lambda \xi \cdot \xi \geq C_{\Lambda}|\xi|^{2}, \forall \xi \in \mathbb{R}^{d}$.

(H2) The fractional flow $f$ is a continuous function satisfies $f(0)=0, f(1)=1$ and $0 \leq f(u) \leq 1$.

(H3) The total mobility $\lambda$ is a continuous function and there exists a positive constant $\lambda_{\star}$, such that $0<\lambda_{\star} \leq \lambda(\cdot)$.

(H4) the capillary pressure term is a continuous function with $\alpha(0)=\alpha(1)=0$.

(H5) The function $\pi: \Sigma_{T} \longrightarrow \mathbb{R}$ is a bounded function in $L^{\infty}\left(0, T ; L^{2}(\partial \Omega)\right)$ with $\int_{\partial \Omega} \pi \mathrm{d} \sigma=0$.

(H6) The initial function $s_{0} \in H^{1}(\Omega)$, and the function $h \in L^{\infty}\left(0, T ; L^{2}(\partial \Omega)\right)$.

In the sequel, we use the Lipschitz continuous nondecreasing function $\beta: \mathbb{R}^{+} \longrightarrow \mathbb{R}^{+}$defined by

$$
\beta(s):=\int_{0}^{s} \alpha(\zeta) \mathrm{d} \zeta, \quad \forall s \in \mathbb{R}^{+} .
$$

We now give the definition of a weak solution of the problem (1).

Definition 1 A weak solution of system (1) is a pair of function $(s, p), s, p: Q_{T} \rightarrow \mathbb{R}$, such that $\forall T>0, s \in$ $W^{1,2}\left(0, T ; H^{1}(\Omega)\right), p \in L^{2}\left(0, T ; H_{M}^{1}(\Omega)\right)$; and for all $\varphi \in L^{2}\left(0, T ; H^{1}(\Omega)\right)$ we have :

$$
\begin{gathered}
\int_{0}^{T} \int_{\Omega} \partial_{t} s \varphi d x d t+\mu \int_{0}^{T} \int_{\Omega} \nabla \partial_{t} s \cdot \nabla \varphi d x d t+\int_{0}^{T} \int_{\Omega} \Lambda f(s) \lambda(s) \nabla p \cdot \nabla \varphi d x d t \\
+\int_{0}^{T} \int_{\Omega} \Lambda \alpha(s) \nabla s \cdot \nabla \varphi d x d t-\int_{0}^{T} \int_{\partial \Omega} h \varphi d x d t=0 \\
\int_{0}^{T} \int_{\Omega} \Lambda \lambda(s) \nabla p \cdot \nabla \varphi d x d t-\int_{0}^{T} \int_{\partial \Omega} \pi \varphi d x d t=0
\end{gathered}
$$

where, $H_{M}^{1}(\Omega)=\left\{u \in H^{1}(\Omega), \int_{\Omega} u=0\right\}$.

Numerical discretization and simulation of multi-phase flow in porous media with Darcy velocity have been the object of several studies during the past decades. There is an extensive literature on the approximation of incompressible two-phase flows with Darcy's velocities for each phase. For instance the finite difference method can be found in the book of Aziz and Settari [23]. The method of finite element methods (mixed or hybrid) is also studied in the past years see Chavent et al. [8] and Chen et al. [9]. For the incompressible immiscible model; let us mention the work of Eymard, Herbin and Michel [15] where a coupled scheme, consisting in a finite volume method together with a phase-by-phase upstream weighting scheme, is analyzed on orthogonal admissible mesh. In [5], the authors propose to explore the limit of a finite volume scheme of two-phase flow model with discontinuous capillary pressure. For that, they proposed to study the limit of the upstream finite volume scheme for the incompressible immiscible model. In [19], the authors suggest a second order accurate finite volume scheme for the Richards equation. Otherwise, the numerical analysis of finite volume and a finite volume-finite element schemes for a compressible two phases flow in porous media for Darcy flow have been studied in the works [3,21].

At our knowledge, the numerical analysis of combined finite volume-finite element scheme of the problem (1) is not studied on triangular mesh. Nevertheless, we mention the pioneer work [11] on the convergence analysis of the finite difference scheme of the Brinkman regularization of two incompressible phase flows in porous media. Recently, in [20], we have been studied the finite volume scheme of Darcy-Brinkman model.

Our aim is to propose a finite volume-finite element scheme based on upstream approach of fractional flow with respect to the gradient of the global pressure.

The rest of this paper is organized as follows. In section 2, we define a primal triangular mesh and its corresponding dual mesh, next, we introduce the nonlinear finite volume-finite element scheme and specify the discretization of the degenerate diffusion and convection terms. In Section 3, we prove the existence of a discrete solution to the finite volume-finite element scheme based on the establishment of a priori estimates on the 
discrete solution. In Section 4, we give estimates on differences of time and space translates for the approximate solutions. Finally, in Section 5, using the Kolmogorov relative compactness criterion, we prove the convergence of a subsequent of discrete solutions to the weak solution in the sense of definition 1 .

\section{Combined Finite volume-Finite element scheme for system (1)}

This section is devoted to the formulation of a combined scheme for the anisotropic Darcy-Brinkman model (1). First, we will describe the space and time discretization, define the approximation spaces and then we will introduce the combined scheme.

\subsection{Space and time discretizations}

In order to discretize problem (1), we perform a finite element triangulation $\left\{\mathcal{T}_{h}\right\}$ of the polygonal domain $\Omega$, consisting of open bounded triangles such that $\bar{\Omega}=\cup_{K \in \mathcal{T}_{h}} \bar{K}$ and such that for all $K, L \in \mathcal{T}_{h}, K \neq L$, then $\bar{K} \cap \bar{L}$ is either an empty set or a common vertex or edge of $K$ and $L$. We denote by $\mathcal{E}_{h}$ the set of all edges, by $\mathcal{E}_{h}^{\text {int }}$ the set of interior edges, by $\mathcal{E}_{h}^{\text {ext }}$ the set of all exterior edges and by $\mathcal{E}_{K}$ the set of all edges of an element $K$. We define $h:=\max \left\{\operatorname{diam}(K), K \in \mathcal{T}_{h}\right\}$ and make the following shape regularity assumption on the family of triangulation $\left\{\mathcal{T}_{h}\right\}_{h}:$

$$
\min _{K \in \mathcal{T}_{h}} \frac{|K|}{(\operatorname{diam}(K))^{d}} \geq k_{\mathcal{T}} \quad \forall h>0 .
$$

Assumption (4) is equivalent to the more common requirement of the existence of a constant $\theta_{\mathcal{T}}>0$ such that

$$
\max _{K \in \mathcal{T}_{h}} \frac{\operatorname{diam}(K)}{\rho_{k}} \geq \theta_{\mathcal{T}} \quad \forall h>0,
$$

where $\rho_{k}$ is the diameter of the largest ball inscribed in the simplex $K$.

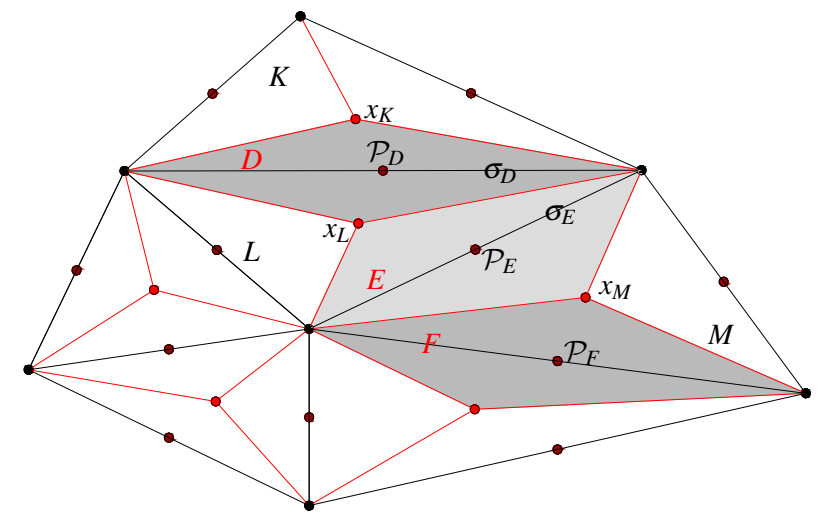

Fig. 1 Triangles $K, L \in \mathcal{T}_{h}$ and dual volumes $D, E \in \mathcal{D}_{h}$ associated with edges $\sigma_{D}, \sigma_{E} \in \mathcal{E}_{h}$.

We also define a dual mesh $\mathcal{D}_{h}$ generated by the triangulation mesh $\mathcal{T}_{h}$ such that $\bar{\Omega}=\cup_{D \in \mathcal{D}_{h}} \bar{D}$. There is one dual element $D$ associated with each side $\sigma_{D}=\sigma_{K, L} \in \mathcal{E}_{h}$ a diamond. We construct it by connecting the barycenters of every $K \in \mathcal{T}_{h}$ that contains $\sigma_{D}$ through the vertices of $\sigma_{D}$. As for the primal mesh, we define $\mathcal{F}_{h}, \mathcal{F}_{h}^{\text {int }}$ and $\mathcal{F}_{h}^{\text {ext }}$, respectively, as the set of dual, interior, and exterior mesh edges. For $\sigma_{D} \in \mathcal{F}_{h}^{e x t}$, the contour of $D$ is completed 
by the edge $\sigma_{D}$ itself which corresponds to a half diamond. We refer to the Fig. 1 for the 2D case.

We use the following notations :

- $|D|=\operatorname{mes}(D)=d$-dimensional Lebesgue measure of $D$ and $|\sigma|$ is the $(d-1)$-dimensional measure of $\sigma$.

- $\mathcal{P}_{D}$ is the barycenter of the edge $\sigma_{D}$.

- $\mathcal{N}(D)$ the set of neighbors of the diamond $D$.

- $d_{D, E}:=\left|\mathcal{P}_{E}-\mathcal{P}_{D}\right|$ the distance between the center $\mathcal{P}_{D}$ and $\mathcal{P}_{E}$.

- $\sigma_{D, E}$ the interface between a dual volume $D$ and $E \in \mathcal{N}(D)$.

- $\eta_{D, E}$ the unit normal vector to $\sigma_{D, E}$ outward to $D$.

- $K_{D, E}=\left\{K \in \mathcal{T}_{h} ; \sigma_{D, E} \subset K\right\}$.

- $\mathcal{D}_{h}^{\text {int }}$ and $\mathcal{D}_{h}^{\text {ext }}$ are, respectively, the set of all interior and boundary dual volumes.

Next, we define the following finite-dimensional space :

$$
X_{h}:=\left\{\varphi_{h} \in L^{2}(\Omega) ;\left.\varphi_{h}\right|_{K} \text { is linear } \forall K \in \mathcal{T}_{h}, \varphi_{h} \text { is continuous at the points } \mathcal{P}_{D}, D \in \mathcal{D}_{h}^{\text {int }}\right\} .
$$

The basis of $X_{h}$ is spanned by the shape functions $\varphi_{D}, D \in \mathcal{D}_{h}$ such that $\varphi_{D}\left(\mathcal{P}_{E}\right)=\delta_{D E}, E \in \mathcal{D}_{h}$ and $\delta$ being the Kronecker symbol. We recall that the approximations in these spaces are nonconforming as $X_{h} \not \subset H^{1}(\Omega)$. We equip $X_{h}$ with the semi-norm,

$$
\|\left. S_{h}\right|_{X_{h}} ^{2}:=\sum_{K \in \mathcal{T}_{h}} \int_{K}\left|\nabla S_{h}\right|^{2} d x
$$

For the time discretization, it's might be performed with a variable time step, to simplify the notation, we consider a constant time step $\Delta t \in[0, T]$. A discretization of $[0, T]$ is given by $N \in \mathbb{N}^{*}$ such that $t_{n}=n \Delta t$, for $n \in\{0, . ., N+1\}$ with $t_{N+1}=T$. For a given value $W_{D}, D \in \mathcal{D}_{h}$ (resp. $W_{D}^{n}, D \in \mathcal{D}_{h}, n \in[0, N]$ ), we define a constant piecewise function as : $w(x)=W_{D}$ for $x \in D\left(\operatorname{resp} . w(t, x)=W_{D}^{n}\right.$ for $\left.\left.\left.x \in D, t \in\right] t_{n}, t_{n+1}\right]\right)$.

\subsection{Combined scheme}

This subsection is committed to discretize the Darcy-Brinkman system (1) with anisotropic tensors on general meshes where we loose the orthogonality condition then the classical approximation of the normal diffusive flux used in the typical finite volume scheme VF4 (see [14] for more details) and therefore the consistency of the scheme. To discretize (1), we use the implicit Euler scheme in time and we consider the piecewise linear nonconforming finite element method for the discretization of the diffusion term in space. The convection terms are discretized by means of a finite volume scheme on the dual mesh.

The approximation of the flux $K \nabla p \cdot \eta_{D, E}$ on the interface $\sigma_{D, E}$ is denoted by $\delta P_{D, E}$. Now, we have to approximate $K f(s) \lambda(s) \nabla p \cdot \eta_{D, E}$ by means of the values $S_{D}, S_{E}$, and $\delta P_{D, E}$ that are available in the neighborhood of the interface $\sigma_{D, E}$. To do this, we use a numerical flux function $G_{s}\left(S_{D}, S_{E}, \delta P_{D, E}\right)$. Numerical convection flux functions $G_{s}$ of arguments $(a, b, c) \in \mathbb{R}^{3}$ are required to satisfy the properties:

- $G_{s}(., b, c)$ is nondecreasing for all $b, c \in \mathbb{R}$, and $G_{s}(a, ., c)$ is nonincreasing for all $a, c \in \mathbb{R}$;

- $G_{s}(a, b, c)=G_{s}(b, a, c)$ for all $a, b, c \in \mathbb{R}$; hence the flux is conservative.

- $G_{s}(a, a, c)=-f(a) \lambda(a) c$ for all $a, c \in \mathbb{R}$; hence the flux is consistent.

One possibility to construct the numerical flux corresponding to $g(s) c$ is by split $g(s)$ to a nondecreasing part $(g(.))_{\uparrow}$ and a nonincreasing part $(g(.))_{\downarrow}$ such that

$$
g_{\uparrow}(z):=\int_{0}^{z}\left(g^{\prime}(s)\right)^{+} d s, \quad g_{\downarrow}(z):=-\int_{0}^{z}\left(g^{\prime}(s)\right)^{-} d s,
$$

herein $\left.\left(g^{\prime}(s)\right)^{+}=\max \left(g^{\prime}(s)\right), 0\right)$ and $\left.\left(g^{\prime}(s)\right)^{-}=\max \left(-g^{\prime}(s)\right), 0\right)$. Then we take, 
FV scheme for Darcy-Brinkman's model of two-phase flows

$$
G_{S}(a, b, c)=c^{+}\left(g_{\uparrow}(a)+g_{\downarrow}(b)\right)-c^{-}\left(g_{\uparrow}(b)+g_{\downarrow}(a)\right) .
$$

In our case $g()=.-f(.) \lambda($.$) and since f \lambda$ is nondecreasing with $f(0)=0$ then,

$$
G_{s}(a, b, c)=-f(b) \lambda(b) c^{+}-(-f(a) \lambda(a)) c^{-} .
$$

Next, for all $S_{h}=\sum_{D \in \mathcal{D}_{h}} S_{D} \varphi_{D}$, we define a discrete function $\beta_{h}\left(S_{h}\right)$ as

$$
\beta_{h}\left(S_{h}\right)=\sum_{D \in \mathcal{D}_{h}} \beta\left(S_{D}\right) \varphi_{D}
$$

Finally, a combined finite volume-nonconforming finite element scheme for the discretization of the problem (1) is given by the following set of equations: For all $D \in D_{h}$,

$$
S_{D}^{0}=\frac{1}{|D|} \int_{D} s_{0}(x) d x
$$

and for all $D \in \mathcal{D}_{h}, n \in\{0,1, \ldots, N\}$,

$$
\begin{gathered}
|D| \frac{S_{D}^{n+1}-S_{D}^{n}}{\Delta t}-\mu \sum_{E \in \mathcal{N}(D)} \mathcal{N}_{D, E}\left(\frac{S_{E}^{n+1}-S_{E}^{n}}{\Delta t}-\frac{S_{D}^{n+1}-S_{D}^{n}}{\Delta t}\right)-\sum_{E \in \mathcal{N}(D)} \mathcal{M}_{E, D}\left(\beta\left(S_{E}^{n+1}\right)-\beta\left(S_{D}^{n+1}\right)\right) \\
+\sum_{E \in \mathcal{N}(D)} G_{S}\left(S_{D}^{n+1}, S_{E}^{n+1} ; \delta P_{D, E}^{n+1}\right)=|\partial D \cap \partial \Omega| h_{D}^{n+1}, \\
-\sum_{E \in \mathcal{N}(D)} G_{p}\left(S_{D}^{n+1}, S_{E}^{n+1} ; \delta P_{D, E}^{n+1}\right)=|\partial D \cap \partial \Omega| \pi_{D}^{n+1}, \\
\sum_{D \in \mathcal{D}_{h}} P_{D}^{n+1} \varphi_{D}=0 . \\
h_{D}^{n+1}=\frac{1}{\Delta t|\partial D \cap \partial \Omega|} \int_{\partial D \cap \partial \Omega} \int_{t_{n}}^{t_{n+1}} h(t, x) d x, \quad \pi_{D}^{n+1}=\frac{1}{\Delta t|\partial D \cap \partial \Omega|} \int_{\partial D \cap \partial \Omega} \int_{t_{n}}^{t_{n+1}} \pi(t, x) d x .
\end{gathered}
$$

The coefficient $\mathcal{N}_{D, E}$ (resp. $\mathcal{M}_{D, E}$ ) for $D, E \in \mathcal{D}_{h}^{\text {int }}$ is the stiffness coefficient of the nonconforming finite element method. So that:

$$
\mathcal{N}_{D, E}=-\sum_{K \in \mathcal{T}_{h}}\left(\nabla \varphi_{E}, \nabla \varphi_{D}\right)_{L^{2}(K)} \quad \text { and } \quad \mathcal{M}_{D, E}=-\sum_{K \in \mathcal{T}_{h}}\left(\Lambda \nabla \varphi_{E}, \nabla \varphi_{D}\right)_{L^{2}(K)}
$$

$\delta P_{D, E}^{n+1}$ denotes the approximation of $K \nabla p$ on the interface $\sigma_{D, E}$ :

$$
\delta P_{D, E}^{n+1}=\mathcal{M}_{D, E}\left(P_{E}^{n+1}-P_{D}^{n+1}\right)
$$

and we define $G_{p}$ for all $a, b, c \in \mathbb{R}$ as

$$
G_{p}(a, b, c)=\lambda(b) c^{+}-\lambda(a) c^{-} .
$$

Notice that the source terms are, for $n \in\{0, \ldots, N\}$

Remark 1 A rigorous justification for the construction of the schema for nonlinear convection-diffusion problems can be found in $[1,13]$ and we only put the formal construction of the schema in this paper.

Remark 2 We can rewrite (7) as follow : 


$$
\begin{aligned}
|D| \frac{S_{D}^{n+1}-S_{D}^{n}}{\Delta t}-\mu \sum_{E \in \mathcal{D}_{h}} \mathcal{N}_{D, E}\left(\frac{S_{E}^{n+1}-S_{E}^{n}}{\Delta t}\right)-\sum_{E \in \mathcal{D}_{h}} & \mathcal{M}_{E, D}\left(\beta\left(S_{E}^{n+1}\right)\right) \\
& +\sum_{E \in \mathcal{N}(D)} G_{s}\left(S_{D}^{n+1}, S_{E}^{n+1} ; \delta P_{D, E}^{n+1}\right)=|\partial D \cap \partial \Omega| h_{D}^{n+1} .
\end{aligned}
$$

Proof. The proof of this assertion is given in Lemma 5 and by equation (21)-(22).

Definition 2 Using the values of $\left(\left(S_{D}^{n+1}, P_{D}^{n+1}\right), \forall D \in \mathcal{D}_{h}\right.$ and $n \in[0, . ., N]$, we will define two approximate solutions of discrete problem (7)-(9) in the sense of the combined finite volume-nonconforming finite element scheme : i) A nonconforming finite element solution $\left(S_{h, \Delta t}, P_{h, \Delta t}\right)$ as a function piecewise linear and continuous at the barycenters of the interior sides in space and piecewise constant in time, such that:

$$
\begin{gathered}
S_{h, \Delta t}(x, 0)=S_{h}^{0}(x) \text { for } x \in \Omega, \\
\left.\left.\left(S_{h, \Delta t}(x, t), P_{h, \Delta t}(x, t)\right)=\left(S_{h}^{n+1}(x), P_{h}^{n+1}(x)\right) \text { for } x \in \Omega, t \in\right] t_{n}, t_{n+1}\right], \\
\text { where } S_{h}^{n+1}=\sum_{D \in \mathcal{D}_{h}} S_{D}^{n+1} \varphi_{D} \quad \text { and } \quad P_{h}^{n+1}=\sum_{D \in \mathcal{D}_{h}} P_{D}^{n+1} \varphi_{D} .
\end{gathered}
$$

ii) A finite volume solution $\left(\tilde{S}_{h, \Delta t}, \tilde{P}_{h, \Delta t}\right)$ defined as piecewise constant on the dual volumes in space and piecewise constant in time, such that:

$$
\begin{gathered}
\tilde{S}_{h, \Delta t}(x, 0)=S_{D}^{0} \text { for } x \in D, D \in \mathcal{D}_{h}, \\
\left.\left.\left(\tilde{S}_{h, \Delta t}(x, t), \tilde{P}_{h, \Delta t}(x, t)\right)=\left(S_{D}^{n+1}, P_{D}^{n+1}\right) \text { for } x \in D, D \in \mathcal{D}_{h}, t \in\right] t_{n}, t_{n+1}\right] .
\end{gathered}
$$

Furthermore, we define a piecewise linear function in time given by

$$
\left.\left.f_{S}(t)=f(S(t, x))=\frac{S\left(t_{n+1}, x\right)-S\left(t_{n}, x\right)}{\Delta t}\left(t-t_{n}\right)+S\left(t_{n}, x\right) \text { for } x \in \Omega, t \in\right] t_{n}, t_{n+1}\right] .
$$

Now, we state the existence theorem under the assumption that all transmissibilities coefficients are positive:

$$
\mathcal{N}_{D, E} \geq 0 \text { and } \mathcal{M}_{D, E} \geq 0, \forall D \in \mathcal{D}_{h}, E \in \mathcal{N}(D) .
$$

Remark 3 Assumption (15) is satisfied when the diffusion term reduces to a scalar function and when the magnitude of all interior angles smaller or equal to $\pi / 2$ in two space dimension.

Theorem 1. Assume $(H 1)-(H 6)$ hold. Under assumption (15), the sequence $\left(S_{h, \Delta t}, P_{h, \Delta t}\right)$ converges to a solution $(s, p)$ of system (1) in the sense of Definition 1 .

The proof of this theorem is splitted in several Lemmas and Propositions in the following section.

\section{Existence and discrete properties}

In this section, we present some technical Lemmas that show the conservativity of the scheme, the coercivity, and the continuity of the diffusion term. We show also a priori estimate on the gradient of the solution which we shall need later in the proof of the existence of a discrete solution of (7)-(9) and in the proof of the convergence.

\subsection{Preliminary results}

This subsection is devoted to known results. For the sake of clarity, we summarize the following lemmas : 
Lemma 1 (Discrete Gronwalls Inequality). Given $\gamma_{1} \geq 0$ and $\gamma_{2} \geq 0$, assume that for all sequence $\left\{y_{n}\right\}_{n}$ satisfies $0 \leq y_{n+1} \leq y_{n}+\gamma_{1} \Delta t+\gamma_{2} \Delta t y_{n+1}$ for all $n \in \mathbb{N}$. Given a fixed time-step $\Delta t_{0} \leq \frac{1}{\gamma_{2}}$ and a fixed time $T>0$, we have for all $\Delta t \leq \Delta t_{0}$,

$$
\forall n \in N, \quad n \Delta t \leq T \Rightarrow y_{n} \leq\left(y_{0}+\frac{\gamma_{1}}{\gamma_{2}}\right) \exp \left(\frac{\gamma_{2} T}{1-\gamma_{2} \Delta t_{0}}\right)
$$

Proof. the proof of this Lemma can be found in [12].

Lemma 2. Let $\Omega \subset \mathbb{R}^{d}\left(d=2\right.$ or $d=3$ ) be an open bounded polygonal with a boundary $\partial \Omega$. Let $\mathcal{T}_{h}$ an admissible mesh. Let $U_{K}$ the value of $u$ on the control volume $K$. Then, there exists a positive constant $C$ depend on $\Omega$ such that :

$$
\|u\|_{L^{2}(\Omega)}^{2} \leq C\|u\|_{X_{h}}^{2}+\frac{4}{|\Omega|}\left(\int_{\Omega} u(x) d x\right)^{2}
$$

Proof. The proof of this Lemma is given in [24, theorem 8.1]

Lemma 3 (Discrete trace lemma). Let $\Omega \subset \mathbb{R}^{d}(d=2$ or $d=3$ ) be an open bounded polygonal with a boundary $\partial \Omega$. Let $\mathcal{T}_{h}$ an admissible mesh. Let $S_{K}$ the value of $s_{h}$ on the control volume $K$. We define $\gamma\left(s_{h}\right)$ by $\gamma\left(s_{h}\right)=S_{K}$ piecewise on $\sigma_{K}=\partial K \cap \partial \Omega$ for all $K \in \mathcal{T}_{h}$. Then, there exists a positive constant $C_{1}$ depend on $\Omega$ such that :

$$
\left\|\gamma\left(s_{h}\right)\right\|_{L^{2}(\partial \Omega)} \leq C_{1}\left(\left\|s_{h}\right\|_{X_{h}}+\left\|s_{h}\right\|_{L^{2}(\Omega)}\right) .
$$

Proof. By applying the trace theorem on the reference simplex and then using a homogeneity/scaling argument (see [4]).

Lemma 4. For all $W_{h}=\sum_{D \in \mathcal{D}_{h}} W_{D} \varphi_{D} \in X_{h}$, we have

$$
\begin{gathered}
\sum_{\sigma_{D, E} \in \mathcal{D}_{h}^{\text {int }}} \operatorname{diam}\left(K_{D, E}\right)^{d-2}\left(W_{E}-W_{D}\right)^{2} \leq \frac{d+1}{2 d k_{\mathcal{T}}}\left\|W_{h}\right\|_{X_{h}}^{2}, \\
\sum_{\sigma_{D, E} \in \mathcal{D}_{h}^{\text {int }}} \frac{\left|\sigma_{D, E}\right|}{d_{D, E}}\left(W_{E}-W_{D}\right)^{2} \leq \frac{d+1}{2(d-1) k_{\mathcal{T}}}\left\|W_{h}\right\|_{X_{h}}^{2} .
\end{gathered}
$$

Proof. This Lemma is proved in [13], but for the sake of completeness we reproduce the proof. We notice that :

$$
d_{D, E} \leq \frac{\operatorname{diam}\left(K_{D, E}\right)}{d},\left|\sigma_{D, E}\right| \leq \frac{\left(\operatorname{diam}\left(K_{D, E}\right)\right)^{d-1}}{d-1} .
$$

then,

$$
\begin{gathered}
\sum_{\sigma_{D, E} \in \mathcal{F}_{h}^{\text {int }}}\left(\operatorname{diam}\left(K_{D, E}\right)\right)^{d-2}\left(W_{E}-W_{D}\right)^{2} \leq\left.\sum_{\sigma_{D, E} \in \mathcal{F}_{h}^{\text {int }}}\left(\operatorname{diam}\left(K_{D, E}\right)\right)^{d-2}\left|\nabla W_{h}\right|_{K_{D, E}}\right|^{2} d_{D, E}^{2} \\
\leq\left.\frac{d+1}{2 d} \sum_{K \in \mathcal{T}_{h}}(\operatorname{diam}(K))^{d}\left|\nabla W_{h}\right|_{K}\right|^{2} \leq\left.\frac{d+1}{2 d k_{\mathcal{T}}} \sum_{K \in \mathcal{T}_{h}}|K|\left|\nabla W_{h}\right|_{K}\right|^{2}=\frac{d+1}{2 d k_{\mathcal{T}}}|| W_{h}||_{X_{h}}^{2} .
\end{gathered}
$$

Using the fact that the gradient of $W_{h}$ is a piecewise constant on $\mathcal{T}_{h}$, the inequality (18) and each $K \in \mathcal{T}_{h}$ contains exactly $\left(\begin{array}{c}d+1 \\ 2\end{array}\right)=\frac{d(d+1)}{2}$ dual sides with assumption (4), we manage to show (16). similarly,

$$
\sum_{\sigma_{D, E} \in \mathcal{F}_{h}^{\text {int }}} \frac{\left|\sigma_{D, E}\right|}{d_{D, E}}\left(W_{E}-W_{D}\right)^{2} \leq\left.\sum_{\sigma_{D, E} \in \mathcal{F}_{h}^{\text {int }}}\left|\nabla W_{h}\right|_{K_{D, E}}\right|^{2} d_{D, E}\left|\sigma_{D, E}\right| \leq\left.\frac{d+1}{2(d-1) k_{\mathcal{T}}}|| W_{h}\right|_{X_{h}} ^{2} .
$$


Lemma 5. For all $D \in \mathcal{D}_{h}$ :

$$
\mathcal{N}_{D, D}=-\sum_{E \in \mathcal{N}(D)} \mathcal{N}_{D, E} \quad \text { and } \quad \mathcal{M}_{D, D}=-\sum_{E \in \mathcal{N}(D)} \mathcal{M}_{D, E} .
$$

Proof. We can find the proof in [13]. for reader convenience we reproduce this proof. We fix a dual volume $D \in \mathcal{D}_{h}$. By using the structure of $\varphi_{D}$, the sum (10) is reduced just for two triangles $K$ and $L$ which have $\sigma_{D}$ as a common interface,

$$
\mathcal{M}_{D, D}=-\left(\Lambda \nabla \varphi_{D}, \nabla \varphi_{D}\right)_{L^{2}(L)}-\left(\Lambda \nabla \varphi_{D}, \nabla \varphi_{D}\right)_{L^{2}(K)} .
$$

We denote by $E_{1}$ and $E_{2}$ the two dual volumes associated of the two other side of element $L$. Since we have

$$
\varphi_{D}+\varphi_{E_{1}}+\varphi_{E_{2}}=1 \text { on } L,
$$

then

$$
-\left(\Lambda \nabla \varphi_{D}, \nabla \varphi_{D}\right)_{L^{2}(L)}=\left(\Lambda \nabla \varphi_{E_{1}}, \nabla \varphi_{D}\right)_{L^{2}(L)}+\left(\Lambda \nabla \varphi_{E_{2}}, \nabla \varphi_{D}\right)_{L^{2}(L)} .
$$

By using a similar eventual contribution for the element $K$ and by replacing that in the equation (20), this yield to prove the first assertion of Lemma 5. The same guidelines are used to prove the second assertion of this Lemma.

Using the fact that $\mathcal{N}_{D, E}=0$ and $\mathcal{M}_{D, E}=0$ unless if $E \in \mathcal{N}(D)$ or if $E=D$, we deduce from (19):

$$
\begin{aligned}
\sum_{E \in \mathcal{D}_{h}} \mathcal{M}_{D, E} \beta\left(M_{E}^{n+1}\right) & =\sum_{E \in \mathcal{N}(D)} \mathcal{M}_{D, E} \beta\left(S_{E}^{n+1}\right)+\mathcal{M}_{D, D} \beta\left(S_{D}^{n+1}\right) \\
& =\sum_{E \in \mathcal{N}(D)} \mathcal{M}_{D, E}\left(\beta\left(S_{E}^{n+1}\right)-\beta\left(S_{D}^{n+1}\right)\right), \\
\sum_{E \in \mathcal{D}_{h}} \mathcal{N}_{D, E} S_{E}^{n+1} & =\sum_{E \in \mathcal{N}(D)} \mathcal{N}_{D, E}\left(S_{E}^{n+1}-S_{D}^{n+1}\right) .
\end{aligned}
$$

Let us take two fixed neighboring dual volumes $D$ and $E \in \mathcal{D}_{h}$. Using the symmetry of the tensor $\Lambda$, we remark that $\mathcal{M}_{D, E}=\mathcal{M}_{E, D}$, which yields to an equality up to the sign between two discrete diffusive flux, from $D$ to $E$ and from $E$ to $D$. In other terms,

$$
\mathcal{M}_{D, E}\left(S_{E}^{n+1}-S_{D}^{n+1}\right)=-\mathcal{M}_{E, D}\left(S_{D}^{n+1}-S_{E}^{n+1}\right) .
$$

Lemma 6 (Coercivity). For all $\beta_{h}\left(S_{h}\right)=\sum_{D \in \mathcal{D}_{h}} \beta\left(S_{D}\right) \varphi_{D}$ and $W_{h}=\sum_{D \in \mathcal{D}_{h}} W_{D} \varphi_{D} \in X_{h}$, then, the discrete degenerate diffusion operator is continuous coercive and we have

$$
\sum_{D \in \mathcal{D}_{h}} \sum_{E \in \mathcal{N}(D)} \mathcal{M}_{D, E}\left(\beta\left(S_{E}\right)-\beta\left(S_{D}\right)\right)^{2} \geq C_{\Lambda}\left\|\beta_{h}\left(S_{h}\right)\right\|_{X_{h}}^{2}, \quad \sum_{D \in \mathcal{D}_{h} E \in \mathcal{N}(D)} \sum_{D, E}\left(S_{E}-S_{D}\right)^{2}=\left\|W_{h}\right\|_{X_{h}}^{2},
$$

and

$$
\left|\sum_{D \in \mathcal{D}_{h}} \sum_{E \in \mathcal{N}(D)} \mathcal{M}_{D, E}\left(\beta\left(S_{E}\right)-\beta\left(S_{D}\right)\right)^{2}\right| \leq C_{\Lambda}^{\prime}\left\|\beta_{h}\left(S_{h}\right)\right\|_{X_{h}}^{2}
$$

Proof. We have

$$
\begin{aligned}
\sum_{D \in \mathcal{D}_{h}} \sum_{E \in \mathcal{N}(D)} \mathcal{M}_{D, E}\left(\beta\left(S_{E}\right)-\beta\left(S_{D}\right)\right)^{2} & =-2 \sum_{D \in \mathcal{D}_{h}} \beta\left(S_{D}\right) \sum_{E \in \mathcal{N}(D)} \mathcal{M}_{D, E}\left(\beta\left(S_{E}\right)-\beta\left(S_{D}\right)\right) \\
& =-2 \sum_{D \in \mathcal{D}_{h}} \beta\left(S_{D}\right) \sum_{E \in \mathcal{D}_{h}} \mathcal{M}_{D, E} \beta\left(S_{E}\right)
\end{aligned}
$$


Then, by using the definition of $\beta_{h}\left(S_{h}\right)$ and (10) we get:

$$
-\sum_{D \in \mathcal{D}_{h}} \beta\left(S_{D}\right) \sum_{E \in \mathcal{D}_{h}} \mathcal{M}_{D, E} \beta\left(S_{E}\right)=\sum_{K \in \mathcal{T}_{h}}\left(\Lambda \nabla \beta_{h}\left(S_{h}\right), \nabla \beta_{h}\left(S_{h}\right)\right)_{L^{2}(K)} .
$$

Now thanks to the coercivity of the tensor $\Lambda$ given by (H2), we can deduce that

$$
\sum_{D \in \mathcal{D}_{h} E \in \mathcal{N}(D)} \mathcal{M}_{D, E}\left(\beta\left(S_{E}\right)-\beta\left(S_{D}\right)\right)^{2} \geq C_{\Lambda} \sum_{K \in \mathcal{T}_{h}}\left(\nabla \beta\left(S_{h}\right), \nabla \beta\left(S_{h}\right)\right)_{L^{2}(K)}
$$

and therefore (24) is a straightforward consequence of the boundedness of the tensor $\Lambda$ given in (H2).

\subsection{A priori estimates}

Proposition 1 Let $\left(S_{D}^{n+1}, P_{D}^{n+1}\right)_{D \in \mathcal{D}_{h}, n \in\{0, \ldots, N\}}$ be a solution of the scheme (7)-(9). Then, there exists a positive constant $C(\Omega)$ depends only on $\Omega$ such that :

$$
\sum_{D \in \mathcal{D}_{h}} \sum_{E \in \mathcal{N}(D)} \mathcal{M}_{E, D}\left(P_{D}^{n+1}-P_{E}^{n+1}\right)^{2} \leq C(\Omega)\|\pi\|_{L^{\infty}\left(0, T ; L^{2}(\partial \Omega)\right)}^{2}
$$

and consequently

$$
\left\|P_{h}^{n+1}\right\|_{X_{h}}^{2} \leq C(\Omega)\|\pi\|_{L^{\infty}\left(0, T ; L^{2}(\partial \Omega)\right)}^{2} .
$$

Proof. We multiply (8) by $P_{D}^{n+1}$, we sum for all $D \in \mathcal{D}_{h}$ and after a discrete integrating by parts we get :

$$
\sum_{D \in \mathcal{D}_{h} \in \in \mathcal{N}(D)} G_{p}\left(S_{D}^{n+1}, S_{E}^{n+1}, \delta P_{D, E}^{n+1}\right)\left(P_{E}^{n+1}-P_{D}^{n+1}\right)=2 \sum_{K \in \mathcal{T}_{h}}|\partial D \cap \partial \Omega| \pi_{D}^{n+1} P_{D}^{n+1}
$$

but the numerical flux satisfies $G_{p}(a, b, c) c \geq \lambda_{*}|c|^{2}$, for all $(a ., b, c) \in \mathbb{R}^{3}$, and under the assumption that the transmissibilities coefficients are positive one gets

$$
\begin{aligned}
\lambda_{*} \sum_{D \in \mathcal{D}_{h}} \sum_{E \in \mathcal{N}(D)} \mathcal{M}_{E, D}\left(P_{E}^{n+1}-P_{D}^{n+1}\right)^{2} & \leq 2 \sum_{D \in \mathcal{D}_{h}}|\partial D \cap \partial \Omega| \pi_{D}^{n+1} P_{D}^{n+1} \\
& \leq \frac{2}{\Delta t} \sum_{D \in \mathcal{D}_{h}}\left(\frac{1}{\beta} \int_{t_{n}}^{t_{n+1}} \int_{\partial D \cap \partial \Omega} \pi^{2} d t d x+4 \beta \Delta t \int_{\partial D \cap \partial \Omega}\left(P_{D}^{n+1}\right)^{2} d x\right)
\end{aligned}
$$

Furthermore, by using the discrete trace lemma and the fact that $\pi \in L^{\infty}\left(0, T ; L^{2}(\partial \Omega)\right)$ we obtain :

$$
\lambda_{*} \sum_{D \in \mathcal{D}_{h} E \in \mathcal{N}(D)} \mathcal{M}_{E, D}\left(P_{E}^{n+1}-P_{D}^{n+1}\right)^{2} \leq \frac{2}{\beta}\|\pi\|_{L^{\infty}\left(0, T ; L^{2}(\partial \Omega)\right)}+\beta C_{1}\left(\left\|P_{h}^{n+1}\right\|_{X_{h}}+\left\|P_{h}^{n+1}\right\|_{L^{2}(\Omega)}\right) .
$$

Applying Lemma 2 to the sequence $\left(P_{h}^{n+1}\right)_{h}$ and using the equation (9), we have

$$
\left\|P_{h}^{n+1}\right\|_{L^{2}(\Omega)} \leq C|| P_{h}^{n+1} \|_{X_{h}},
$$

then from above inequality, we get

$$
\lambda_{*} \sum_{D \in \mathcal{D}_{h}} \sum_{E \in \mathcal{N}(D)} \mathcal{M}_{E, D}\left(P_{E}^{n+1}-P_{D}^{n+1}\right)^{2} \leq \frac{2}{\beta}\|\pi\|_{L^{\infty}\left(0, T ; L^{2}(\partial \Omega)\right)}+\beta C_{1}(1+C)\left\|P_{h}^{n+1}\right\|_{X_{h}} .
$$


Finally, The result follow from the use of the Lemmas 6 and after choosing appropriate parameter $\beta$.

Proposition 2 Let $\left(S_{D}^{n+1}, P_{D}^{n+1}\right)_{D \in \mathcal{D}_{h}, n \in\{0, \ldots, N\}}$ be a solution of the scheme (7)-(9). then, there exists a positive constant $C_{1}(\Omega)$ depends only on $\Omega$, where for all $n \in \mathbb{N}, 0<\Delta t \leq \Delta t_{0}$ and $n \Delta t \leq T$ with $\Delta t_{0} \leq \frac{\mu}{C_{1}(\Omega)}$ we have:

$$
\sum_{D \in \mathcal{D}_{h}}|D|\left|S_{D}^{n}\right|^{2}+\sum_{D \in \mathcal{D}_{h}} \sum_{E \in \mathcal{N}(D)} \mathcal{N}_{E, D}\left|S_{E}^{n}-S_{L}^{n}\right|^{2} \leq g\left(s_{0}, h, \pi, \Delta t_{0}\right)
$$

where

$g=\left(\sum_{D \in \mathcal{D}_{h}}|D|\left|S_{D}^{0}\right|^{2}+\sum_{D \in \mathcal{D}_{h}} \sum_{E \in \mathcal{N}(D)} \mathcal{N}_{E, D}\left|S_{E}^{0}-S_{D}^{0}\right|^{2}+\left\|\left.h\right|_{L^{\infty}\left(0, T ; L^{2}(\partial \Omega)\right)} ^{2}+\right\| \pi \|_{L^{\infty}\left(0, T ; L^{2}(\partial \Omega)\right)}^{2}\right) \exp \left(\frac{C_{1}(\Omega) T}{\mu-C_{1}(\Omega) \Delta t_{0}}\right)$.

Proof. We multiply (7) by $S_{D}^{n+1}$ for all $D \in \mathcal{D}_{h}$, that gives :

$$
\begin{aligned}
& \sum_{D \in \mathcal{D}_{h}} \mid D \mid \frac{S_{D}^{n+1}-S_{D}^{n}}{\Delta t} S_{D}^{n+1}-\mu \sum_{D \in \mathcal{D}_{h} E \in \mathcal{N}(D)} \sum_{E, D}\left(\frac{S_{E}^{n+1}-S_{E}^{n}}{\Delta t}-\frac{S_{D}^{n+1}-S_{D}^{n}}{\Delta t}\right) S_{D}^{n+1} \\
&+\sum_{D \in \mathcal{D}_{h}} \sum_{E \in \mathcal{N}(D)} G_{s}\left(S_{D}^{n+1}, S_{E}^{n+1}, \delta P_{D, E}^{n+1}\right) S_{D}^{n+1}-\sum_{D \in \mathcal{D}_{h} E \in \mathcal{N}(D)} \sum_{E, D}\left(\beta\left(S_{E}^{n+1}\right)-\beta\left(S_{D}^{n+1}\right)\right) S_{D}^{n+1} \\
&=\sum_{D \in \mathcal{D}_{h}}|\partial D \cap \partial \Omega| h_{D}^{n+1} S_{D}^{n+1} .
\end{aligned}
$$

Making an integration by parts and using the inequality $\left[(a-b) a \geq \frac{1}{2}\left(a^{2}-b^{2}\right)\right]$, and using the conservative property of the function $G_{s}$ we obtain

$$
\begin{aligned}
\frac{1}{2} \sum_{D \in \mathcal{D}_{h}}|D| \frac{\left|S_{D}^{n+1}\right|^{2}-\mid}{\Delta t} & \left|S_{D}^{n}\right|^{2} \\
& +\frac{\mu}{4 \Delta t} \sum_{D \in \mathcal{D}_{h}} \sum_{E \in \mathcal{N}(D)} \mathcal{N}_{E, D}\left(\left|S_{E}^{n+1}-S_{D}^{n+1}\right|^{2}-\left|S_{E}^{n}-S_{D}^{n}\right|^{2}\right) \\
& \sum_{D \in \mathcal{D}_{h}} \sum_{E \in \mathcal{N}(D)} \mathcal{M}_{E, D}\left(\beta\left(S_{E}^{n+1}\right)-\beta\left(S_{D}^{n+1}\right)\right)\left(S_{E}^{n+1}-S_{D}^{n+1}\right) \leq \\
& \frac{1}{2} \sum_{D \in \mathcal{D}_{h}} \sum_{E \in \mathcal{N}(D)} G_{S}\left(S_{D}^{n+1}, S_{E}^{n+1}, \delta P_{E, D}^{n+1}\right)\left(S_{E}^{n+1}-S_{D}^{n+1}\right)+\sum_{D \in \mathcal{D}_{h}}|\partial D \cap \partial \Omega| h_{D}^{n+1} S_{D}^{n+1} .
\end{aligned}
$$

The function $\beta$ is a nondecreasing and since $\mathcal{M}_{E, D} \geq 0$, then the diffusion term leads

$$
\frac{1}{2} \sum_{D \in \mathcal{D}_{h}} \sum_{E \in \mathcal{N}(D)} \mathcal{M}_{E, D}\left(\beta\left(S_{E}^{n+1}\right)-\beta\left(S_{D}^{n+1}\right)\right)\left(S_{E}^{n+1}-S_{D}^{n+1}\right) \geq 0
$$

Notice that from (5), we have $\left.\left|G_{s}\left(S_{D}^{n+1}, S_{E}^{n+1}, \delta P_{D, E}^{n+1}\right)\right| \leq C_{1} \mid \delta P_{D, E}^{n+1}\right) \mid$, then the first term in the right hand side of (27), is estimated as

$$
\begin{aligned}
\frac{1}{2} \sum_{D \in \mathcal{D}_{h}} \sum_{E \in \mathcal{N}(D)} G_{s}\left(S_{D}^{n+1}, S_{E}^{n+1}, \delta P_{D, E}^{n+1}\right)\left(S_{E}^{n+1}-S_{D}^{n+1}\right) \leq \\
C_{1} \sum_{D \in \mathcal{D}_{h}} \sum_{E \in \mathcal{N}(D)} \mathcal{M}_{E, D}\left(P_{E}^{n+1}-P_{D}^{n+1}\right)^{2}+C_{1} \sum_{D \in \mathcal{D}_{h} E \in \mathcal{N}(D)} \mathcal{M}_{E, D}\left(S_{E}^{n+1}-S_{D}^{n+1}\right)^{2} .
\end{aligned}
$$

But, according to Lemma 6 if we replace $\beta$ by identity function, we obtain 
FV scheme for Darcy-Brinkman's model of two-phase flows

$$
\sum_{D \in \mathcal{D}_{h} E \in \mathcal{N}(D)} \mathcal{M}_{E, D}\left(S_{E}^{n+1}-S_{D}^{n+1}\right)^{2} \leq C_{\Lambda}\left\|S_{h}\right\|_{X_{h}}=C_{\Lambda} \sum_{D \in \mathcal{D}_{h}} \sum_{E \in \mathcal{N}(D)} \mathcal{N}_{E, D}\left(S_{E}^{n+1}-S_{D}^{n+1}\right)^{2}
$$

then

$$
\begin{aligned}
\frac{1}{2} \sum_{D \in \mathcal{D}_{h}} \sum_{E \in \mathcal{N}(D)} G_{s}\left(S_{D}^{n+1}, S_{E}^{n+1}, \delta P_{D, E}^{n+1}\right)\left(S_{E}^{n+1}-S_{D}^{n+1}\right) & \leq \\
& C_{1} C(\Omega)\|\pi\|_{L^{\infty}\left(0, T ; L^{2}(\partial \Omega)\right)}+C_{\Lambda} \sum_{D \in \mathcal{D}_{h} \in \in \mathcal{N}(D)} \sum_{E, D}\left(S_{E}^{n+1}-S_{D}^{n+1}\right)^{2} .
\end{aligned}
$$

Using the Cauchy-Schwarz inequality with the discrete trace lemma 3 give us

$$
\sum_{D \in \mathcal{D}_{h}}|\partial D \cap \partial \Omega| h_{D}^{n+1} S_{D}^{n+1} \leq \|\left. h\right|_{L^{\infty}\left(0, T ; L^{2}(\partial \Omega)\right)}+C_{2}\left(\sum_{D \in \mathcal{D}_{h}}|D|\left(\left|S_{D}^{n+1}\right|^{2}+\sum_{D \in \mathcal{D}_{h}} \sum_{E \in \mathcal{N}(D)} \mathcal{N}_{E, D}\left|S_{E}^{n+1}-S_{D}^{n+1}\right|^{2}\right)\right.
$$

where $C_{2}$ is a positive constant depend only on $\Omega$. This leads

$$
\begin{aligned}
& \sum_{D \in \mathcal{D}_{h}}|D|\left(\left|S_{D}^{n+1}\right|^{2}-\left|S_{D}^{n}\right|^{2}\right)+\sum_{D \in \mathcal{D}_{h}} \sum_{E \in \mathcal{N}(D)} \mathcal{N}_{E, D}\left(\left|S_{E}^{n+1}-S_{D}^{n+1}\right|^{2}-\left|S_{E}^{n}-S_{D}^{n}\right|^{2}\right) \\
& \leq \frac{4 \Delta t}{\mu}\left(C_{2} \sum_{D \in \mathcal{D}_{h}}|D|\left(S_{D}^{n+1}\right)^{2}+\left(C_{2}+C_{\Lambda}\right) \sum_{D \in \mathcal{D}_{h}} \sum_{E \in \mathcal{N}(D)} \mathcal{N}_{E, D}\left(S_{E}^{n+1}-S_{D}^{n+1}\right)^{2}\right. \\
&\left.+\|h\|_{L^{\infty}\left(0, T ; L^{2}(\partial \Omega)\right)}^{2}+C_{1} C(\Omega)\|\pi\|_{L^{\infty}\left(0, T ; L^{2}(\partial \Omega)\right)}\right),
\end{aligned}
$$

to end this proof, we take $C_{1}(\Omega)=\frac{1}{4} \max \left\{C_{2}, C_{2}+C_{\Lambda}, C_{1} C(\Omega), 1\right\}$ and applying the discrete Gronwall lemma 1 with $y_{n+1}=\sum_{D \in \mathcal{D}_{h}}|D|\left|S_{D}^{n+1}\right|^{2}+\sum_{D \in \mathcal{D}_{h}} \sum_{E \in \mathcal{N}(D)} \mathcal{N}_{E, D}\left|S_{E}^{n+1}-S_{D}^{n+1}\right|^{2}, \gamma_{2}=\frac{C_{1}(\Omega)}{\mu}$ and $\gamma_{1}=\frac{C_{1}(\Omega)}{\mu}\left(\|h\|_{L^{\infty}\left(0, T ; L^{2}(\partial \Omega)\right)}^{2}+\|\pi\|_{L^{\infty}\left(0, T ; L^{2}(\partial \Omega)\right)}\right)$ to get (26).

Proposition 3 Let $\left(S_{D}^{n+1}, P_{D}^{n+1}\right)_{D \in \mathcal{D}_{h}, n \in\{0, \ldots, N\}}$ be a solution of the scheme (7)-(9). Then, there exist a positive constants $C_{2}$ depend only on $\mu$ such that :

$$
\sum_{D \in \mathcal{D}_{h}}|D|\left(\frac{S_{D}^{n+1}-S_{D}^{n}}{\Delta t}\right)^{2}+\sum_{D \in \mathcal{D}_{h}} \sum_{E \in \mathcal{N}(D)} \mathcal{N}_{E, D}\left(\frac{S_{E}^{n+1}-S_{E}^{n}}{\Delta t}-\frac{S_{D}^{n+1}-S_{D}^{n}}{\Delta t}\right)^{2} \leq C_{2}(\mu) g\left(s_{0}, h, \pi, \Delta t_{0}\right) .
$$

In addition, we have

$$
\sum_{n=0}^{N} \Delta t|| \beta_{h}\left(S_{h}^{n+1}\right) \|_{X_{h}}^{2} \leq C
$$

Proof. We multiply (7) by $\frac{S_{D}^{n+1}-S_{D}^{n}}{\Delta t}, D \in \mathcal{D}_{h}$, sum over all and $D \in \mathcal{D}_{h}$, and after integrating by parts we obtain $J_{1}+J_{2}+J_{3}=J_{4}$ where 


$$
\begin{aligned}
& J_{1}=\sum_{D \in \mathcal{D}_{h}}|D|\left(\frac{S_{D}^{n+1}-S_{D}^{n}}{\Delta t}\right)^{2}+\frac{\mu}{2} \sum_{D \in \mathcal{D}_{h}} \sum_{E \in \mathcal{N}(D)} \mathcal{N}_{E, D}\left(\frac{S_{E}^{n+1}-S_{E}^{n}}{\Delta t}-\frac{S_{D}^{n+1}-S_{D}^{n}}{\Delta t}\right)^{2}, \\
& J_{2}=-\frac{1}{2} \sum_{D \in \mathcal{D}_{h}} \sum_{E \in \mathcal{N}(D)} G_{s}\left(S_{D}^{n+1}, S_{E}^{n+1}, \delta P_{D, E}^{n+1}\right)\left(\frac{S_{E}^{n+1}-S_{E}^{n}}{\Delta t}-\frac{S_{D}^{n+1}-S_{D}^{n}}{\Delta t}\right), \\
& J_{3}=\frac{1}{2} \sum_{D \in \mathcal{D}_{h}} \sum_{E \in \mathcal{N}(D)} \mathcal{M}_{E, D}\left(\beta\left(S_{E}^{n+1}\right)-\beta\left(S_{D}^{n+1}\right)\right)\left(\frac{S_{E}^{n+1}-S_{E}^{n}}{\Delta t}-\frac{S_{D}^{n+1}-S_{D}^{n}}{\Delta t}\right), \\
& J_{4}=\sum_{D \in \mathcal{D}_{h}}|\partial D \cap \partial \Omega| h_{D}^{n+1} \frac{S_{D}^{n+1}-S_{D}^{n}}{\Delta t} .
\end{aligned}
$$

First, to estimate the second term $J_{2}$, let us recall that there exist a positive constant $C$, such that $\left.\left|G_{s}\left(S_{D}^{n+1}, S_{E}^{n+1}, \delta P_{D, E}^{n+1}\right)\right| \leq C \mid \delta P_{D, E}^{n+1}\right) \mid$, and by using Cauchy-Schwartz inequality we obtain, for all $\gamma>0$

$$
J_{2} \leq \frac{C}{\gamma} \sum_{D \in \mathcal{D}_{h}} \sum_{E \in \mathcal{N}(D)} \mathcal{M}_{E, D}\left(P_{E}^{n+1}-P_{E}^{n+1}\right)^{2}+\gamma \sum_{D \in \mathcal{D}_{h}} \sum_{E \in \mathcal{N}(D)} \mathcal{M}_{E, D}\left(\frac{S_{E}^{n+1}-S_{E}^{n}}{\Delta t}-\frac{S_{D}^{n+1}-S_{D}^{n}}{\Delta t}\right)^{2}
$$

from the estimate (25) and Lemma6, one gets

$$
J_{2} \leq \frac{C}{\gamma}\|\pi\|_{L^{\infty}\left(0, T ; L^{2}(\partial \Omega)\right)}^{2}+C_{1} \gamma \sum_{D \in \mathcal{D}_{h}} \sum_{E \in \mathcal{N}(D)} \mathcal{N}_{E, D}\left(\frac{S_{E}^{n+1}-S_{E}^{n}}{\Delta t}-\frac{S_{D}^{n+1}-S_{D}^{n}}{\Delta t}\right)^{2} .
$$

Next, we use again Cauchy-Schwartz inequality with the proprieties of $\beta$ to obtain for all $\delta>0$ :

$$
J_{3} \leq \frac{C_{2}}{2 \delta} \sum_{D \in \mathcal{D}_{h}} \sum_{E \in \mathcal{N}(D)} \mathcal{M}_{E, D}\left(S_{E}^{n+1}-S_{D}^{n+1}\right)^{2}+\frac{\delta}{2} \sum_{D \in \mathcal{D}_{h}} \sum_{E \in \mathcal{N}(D)} \mathcal{M}_{E, D}\left(\frac{S_{E}^{n+1}-S_{E}^{n}}{\Delta t}-\frac{S_{D}^{n+1}-S_{D}^{n}}{\Delta t}\right)^{2} .
$$

this yield us by Proposition 2 and Lemma 6 to have

$$
J_{3} \leq \frac{C_{1} C_{2}}{2 \delta} g\left(s_{0}, h, \pi, \Delta t_{0}\right)+\frac{C_{1} \delta}{2} \sum_{D \in \mathcal{D}_{h}} \sum_{E \in \mathcal{N}(D)} \mathcal{N}_{E, D}\left(\frac{S_{E}^{n+1}-S_{E}^{n}}{\Delta t}-\frac{S_{D}^{n+1}-S_{D}^{n}}{\Delta t}\right)^{2} .
$$

Similarly, we have for all $\zeta>0$

$$
J_{4} \leq \frac{C_{3}}{\zeta}\|h\|_{L^{\infty}\left(0, T ; L^{2}(\partial \Omega)\right)}^{2}+\zeta C\left[\sum_{D \in \mathcal{D}_{h}}|D|\left(\frac{S_{D}^{n+1}-S_{D}^{n}}{\Delta t}\right)^{2}+\sum_{D \in \mathcal{D}_{h}} \sum_{E \in \mathcal{N}(D)} \mathcal{N}_{E, D}\left(\frac{S_{E}^{n+1}-S_{E}^{n}}{\Delta t}-\frac{S_{D}^{n+1}-S_{D}^{n}}{\Delta t}\right)^{2}\right] .
$$

Finally, if we chose $\gamma, \delta$ and $\zeta$ small enough, we obtain

$$
\sum_{D \in \mathcal{D}_{h}}|D|\left(\frac{S_{D}^{n+1}-S_{D}^{n}}{\Delta t}\right)^{2}+\sum_{D \in \mathcal{D}_{h} E \in \mathcal{N}(D)} \sum_{E, D}\left(\frac{S_{E}^{n+1}-S_{E}^{n}}{\Delta t}-\frac{S_{D}^{n+1}-S_{D}^{n}}{\Delta t}\right)^{2} \leq C_{4} \mu g\left(s_{0}, h, \pi, \Delta t_{0}\right) .
$$

Now, we are concerned with estimate (29), for that we multiply (7) by $\Delta t \beta\left(S_{D}^{n+1}\right)$ and we sum for all $D \in \mathcal{D}_{h}$ and $n=\{0, \ldots, N\}$ to obtain $E_{1}+E_{2}+E_{3}+E_{4}=E_{5}$, where $\left(E_{i}\right)_{i=1.5}$ are explicitly given and estimate in what follow. According to the convexity of the function $\Upsilon(s)=\int_{0}^{s} \beta(z) d z$, since $\Upsilon^{\prime \prime}(s)=\alpha(s) \geq 0$, we shall have the following inequality : $(a-b) \beta(a) \geq \Upsilon(a)-\Upsilon(b)$. Then, 
FV scheme for Darcy-Brinkman's model of two-phase flows

$$
E_{1}=\sum_{n=0}^{N} \sum_{D \in \mathcal{D}_{h}}|D|\left(S_{D}^{n+1}-S_{D}^{n}\right) \beta\left(S_{D}^{n+1}\right) \geq \sum_{n=0}^{N} \sum_{D \in \mathcal{D}_{h}}|D|\left(\Upsilon\left(S_{D}^{n+1}\right)-\Upsilon\left(S_{D}^{n}\right)\right)=\sum_{D \in \mathcal{D}_{h}}|D|\left(\Upsilon\left(S_{D}^{N}\right)-\Upsilon\left(S_{D}^{0}\right)\right) .
$$

Using of Young inequality, an integrating by parts and the positivity of transmissibilities coefficient $\mathcal{M}_{E, D}$ we obtain for all $\delta>0$

$$
\begin{aligned}
& E_{2}=-\mu \sum_{n=0}^{N} \Delta t \sum_{D \in \mathcal{D}_{h}} \sum_{E \in \mathcal{N}(D)} \mathcal{M}_{E, D}\left(\frac{S_{E}^{n+1}-S_{E}^{n}}{\Delta t}-\frac{S_{D}^{n+1}-S_{D}^{n}}{\Delta t}\right) \beta\left(S_{D}^{n+1}\right) \\
\leq & \frac{\mu}{\delta} \sum_{n=0}^{N} \Delta t \sum_{D \in \mathcal{D}_{h}} \sum_{E \in \mathcal{N}(D)} \mathcal{M}_{E, D}\left(\frac{S_{E}^{n+1}-S_{E}^{n}}{\Delta t}-\frac{S_{D}^{n+1}-S_{D}^{n}}{\Delta t}\right)^{2}+\delta \mu \sum_{n=0}^{N} \Delta t \sum_{D \in \mathcal{D}_{h}} \sum_{E \in \mathcal{N}(D)} \mathcal{M}_{E, D}\left(\beta\left(S_{D}^{n+1}\right)-\beta\left(S_{E}^{n+1}\right)\right)^{2},
\end{aligned}
$$

this yield to

$$
E_{2}=\leq \frac{C \mu}{\delta} g\left(s_{0}, h, \pi, \Delta t_{0}\right)+\delta \mu \sum_{n=0}^{N} \Delta t \sum_{D \in \mathcal{D}_{h}} \sum_{E \in \mathcal{N}(D)} \mathcal{M}_{E, D}\left(\beta\left(S_{D}^{n+1}\right)-\beta\left(S_{E}^{n+1}\right)\right)^{2}
$$

According to equation (23), we have

$$
\begin{aligned}
E_{3} & =-\sum_{n=0}^{N} \Delta t \sum_{D \in \mathcal{D}_{h}} \sum_{E \in \mathcal{N}(D)} \mathcal{M}_{E, D}\left(\beta\left(S_{E}^{n+1}\right)-\beta\left(S_{D}^{n+1}\right)\right) \beta\left(S_{D}^{n+1}\right) \\
& =\frac{1}{2} \sum_{n=0}^{N} \Delta t \sum_{D \in \mathcal{D}_{h}} \sum_{E \in \mathcal{N}(D)} \mathcal{M}_{E, D}\left(\beta\left(S_{E}^{n+1}\right)-\beta\left(S_{D}^{n+1}\right)\right)^{2} \geq C_{\Lambda} \sum_{n=0}^{N} \Delta t\left\|\beta_{h}\left(S_{h}^{n+1}\right)\right\|_{X_{h}}^{2} .
\end{aligned}
$$

Next, we have

$$
E_{4}=\sum_{n=0}^{N-1} \Delta t \sum_{D \in \mathcal{D}_{h}} \sum_{E \in \mathcal{N}(D)} G_{s}\left(S_{D}^{n+1}, S_{E}^{n+1}, \delta P_{D, E}^{n+1}\right) \beta\left(S_{D}^{n+1}\right)
$$

Integrating by part, using $\left.\left|G_{s}\left(S_{D}^{n+1}, S_{E}^{n+1}, \delta P_{D, E}^{n+1}\right)\right| \leq C \mid \delta P_{D, E}^{n+1}\right) \mid$ and the Cauchy-Schwarz inequality, give us

$$
E_{4} \leq \frac{1}{\delta} \sum_{n=0}^{N} \Delta t \sum_{D \in \mathcal{D}_{h}} \sum_{E \in \mathcal{N}(D)} \mathcal{M}_{E, D}\left(P_{E}^{n+1}-P_{D}^{n+1}\right)^{2}+\delta \sum_{n=0}^{N} \Delta t \sum_{D \in \mathcal{D}_{h}} \sum_{E \in \mathcal{N}(D)} \mathcal{M}_{E, D}\left(\beta\left(S_{E}^{n+1}\right)-\beta\left(S_{D}^{n+1}\right)\right)^{2},
$$

for any $\delta>0$. The estimate (25) and Lemma 6 lead to

$$
E_{4} \leq \frac{C}{\delta}\|\pi\|_{L^{\infty}\left(0, T ; L^{2}(\partial \Omega)\right)}^{2}+\delta \sum_{n=0}^{N} \Delta t\left\|\beta_{h}\left(S_{h}\right)\right\|_{X_{h}}^{2}
$$

Finally, we use the continuity of $\beta$ and lemma 3 to obtain for all $\delta>0$

$$
\begin{aligned}
E_{5} & =\sum_{n=0}^{N} \Delta t \sum_{D \in \mathcal{D}_{h}}|\partial D \cap \partial \Omega| h_{D}^{n+1} \beta\left(S_{D}^{n+1}\right) \\
& \leq \frac{C}{\delta}\|h(t, \cdot)\|_{L^{\infty}\left(0, T ; L^{2}(\partial \Omega)\right)}^{2}+\delta C_{1}\left(\sum_{n=0}^{N} \Delta t \sum_{D \in \mathcal{D}_{h}}|D|\left|S_{D}^{n}\right|^{2}+\sum_{n=0}^{N} \Delta t\left\|\beta_{h}\left(S_{h}\right)\right\|_{X_{h}}^{2}\right) \\
& \leq C^{\prime} g\left(s_{0}, h, \pi, \Delta t_{0}\right)+\delta C_{1} \sum_{n=0}^{N} \Delta t\left\|\beta_{h}\left(S_{h}\right)\right\|_{X_{h}}^{2} .
\end{aligned}
$$


In summarize, from the equation $E_{1}+E_{2}+E_{3}+E_{4}=E_{5}$ and by choosing an appropriate $\delta$ we have

$$
\sum_{n=0}^{N} \Delta t|| \beta_{h}\left(S_{h}^{n+1}\right) \|_{X_{h}}^{2} \leq C^{\prime \prime} g\left(s_{0}, h, \pi, \Delta t_{0}\right)+\sum_{D \in \mathcal{D}_{h}}|D| \Upsilon\left(S_{D}^{0}\right) \leq C,
$$

where $C^{\prime \prime}$ and $C$ are positives constants.

\subsection{Existence of discrete solution}

The existence of a discrete solution for the combined scheme is given in the following proposition.

Proposition 4 There exists a least one solution of problem (7)-(9).

Proof. First, we will use the following notation :

$$
\mathcal{M}:=\operatorname{Card}\left(\mathcal{D}_{h}\right), \quad s_{\mathcal{M}}:=\left\{s_{D}^{n+1}\right\}_{D \in \mathcal{D}_{h}} \in \mathbb{R}^{\mathcal{M}}, \quad p_{\mathcal{M}}:=\left\{p_{D}^{n+1}\right\}_{D \in \mathcal{D}_{h}} \in \mathbb{R}^{\mathcal{M}} .
$$

We define the application $\quad \mathcal{P}_{h}: \mathbb{R}^{\mathcal{M}} \times \mathbb{R}^{\mathcal{M}} \mapsto \mathbb{R}^{\mathcal{M}} \times \mathbb{R}^{\mathcal{M}}$

$$
\mathcal{P}_{h}\left(s_{\mathcal{M}}, p_{\mathcal{M}}\right)=\left(\left\{\mathcal{P}_{1, D}\right\}_{D \in \mathcal{D}_{h}},\left\{\mathcal{P}_{2, D}\right\}_{D \in \mathcal{D}_{h}}\right),
$$

with

$$
\begin{aligned}
\mathcal{P}_{1, K}= & |D| \frac{S_{D}^{n+1}-S_{D}^{n}}{\Delta t}-\mu \sum_{D \in \mathcal{D}_{h}} \sum_{E \in \mathcal{N}(D)} \mathcal{N}_{D, E}\left(\frac{S_{E}^{n+1}-S_{E}^{n}}{\Delta t}-\frac{S_{D}^{n+1}-S_{D}^{n}}{\Delta t}\right) \\
& -\sum_{D \in \mathcal{D}_{h}} \sum_{E \in \mathcal{N}(D)} \mathcal{M}_{E, D}\left(\beta\left(S_{E}^{n+1}\right)-\beta\left(S_{D}^{n+1}\right)\right)+\sum_{E \in \mathcal{N}(D)} G_{s}\left(S_{D}^{n+1}, S_{E}^{n+1} ; \delta P_{D, E}^{n+1}\right)-|\partial D \cap \partial \Omega| h_{D}^{n+1} \\
\mathcal{P}_{2, K}= & -\sum_{E \in \mathcal{N}(D)} G_{p}\left(S_{D}^{n+1}, S_{E}^{n+1} ; \delta P_{D, E}^{n+1}\right)-|\partial D \cap \partial \Omega| \pi_{D}^{n+1} .
\end{aligned}
$$

The scalar product of $\left(s_{\mathcal{M}}, p_{\mathcal{M}}\right)$ by the equation (30), using the estimates (25)-(26) and Cauchy-Schwarz inequality permit to get

$$
\left[\mathcal{P}_{h}\left(s_{\mathcal{M}}, p_{\mathcal{M}}\right),\left(s_{\mathcal{M}}, p_{\mathcal{M}}\right)\right] \geq \frac{C_{1}}{\Delta t} \sum_{D \in \mathcal{D}_{h}}|D|\left|S_{D}^{n+1}\right|^{2}+C_{2} \sum_{D \in \mathcal{D}_{h}}|D|\left|P_{D}^{n+1}\right|^{2}-\frac{1}{2 \Delta t} \sum_{D \in \mathcal{D}_{h}}|D|\left|S_{K}^{n}\right|^{2}-C_{3} g\left(s_{0}, h, \pi, \Delta t_{0}\right)
$$

where $C_{1}, C_{2}$ and $C_{3}$ are positives constants, this yields to

$$
\left[\mathcal{P}_{h}\left(s_{\mathcal{M}}, p_{\mathcal{M}}\right),\left(s_{\mathcal{M}}, p_{\mathcal{M}}\right)\right]>0
$$

For $\left|\left(s_{\mathcal{M}}, p_{\mathcal{M}}\right)\right|_{\mathbb{R}_{\mathcal{M}}}$ large enough. And therefore we obtain the existence of $\left(s_{\mathcal{M}}, p_{\mathcal{M}}\right)$ such that $\mathcal{P}_{h}\left(s_{\mathcal{M}}, p_{\mathcal{M}}\right)=$ 0 . Indeed, reasoning by contradiction. Assume that there is no $\left(s_{\mathcal{M}}, p_{\mathcal{M}}\right)$ such that $\mathcal{P}_{h}\left(s_{\mathcal{M}}, p_{\mathcal{M}}\right)=0$. In this case, one can define on a ball of origin center and radius $k$, the following application: $A: \bar{B}(0, k) \rightarrow \bar{B}(0, k)$ which give for each $\left(s_{\mathcal{M}}, p_{\mathcal{M}}\right), A\left(\left(s_{\mathcal{M}}, p_{\mathcal{M}}\right)\right)=-k \frac{\mathcal{P}_{h}\left(s_{\mathcal{M}}, p_{\mathcal{M}}\right)}{\left|\left(s_{\mathcal{M}}, p_{\mathcal{M}}\right)\right|}$, where the constant $k>0$. The map $A$ is continuous due to the continuity of $\mathcal{P}_{h},\left|\mathcal{P}_{h}\left(s_{\mathcal{M}}, p_{\mathcal{M}}\right)\right| \neq 0$ over $\bar{B}(0, k)$ and $\bar{B}(0, k)$ is closed convex and compact. The Brower fixed point theorem implies then that there exists $\left(s_{\mathcal{M}}, p_{\mathcal{M}}\right)$ in $\bar{B}(0, k)$ such that $-k \frac{\mathcal{P}_{h}\left(s_{\mathcal{M}}, p_{\mathcal{M}}\right)}{\left|\left(s_{\mathcal{M}}, p_{\mathcal{M}}\right)\right|}=\left(s_{\mathcal{M}}, p_{\mathcal{M}}\right)$, If we take the norm of both sides of this equation, we see that $\left|\left(s_{\mathcal{M}}, p_{\mathcal{M}}\right)\right|=k>0$ and if we take the scalar product of each side with $\left(s_{\mathcal{M}}, p_{\mathcal{M}}\right)$, we find $\left[\left(s_{\mathcal{M}}, p_{\mathcal{M}}\right),\left(s_{\mathcal{M}}, p_{\mathcal{M}}\right)\right]=\left|\left(s_{\mathcal{M}}, p_{\mathcal{M}}\right)\right|^{2}=-k \frac{\left[\mathcal{P}_{h}\left(s_{\mathcal{M}}, p_{\mathcal{M}}\right),\left(s_{\mathcal{M}}, p_{\mathcal{M}}\right)\right]}{\left|\left(s_{\mathcal{M}}, p_{\mathcal{M}}\right)\right|} \leq 0$, this yield to contradiction. 
FV scheme for Darcy-Brinkman's model of two-phase flows

\section{Convergence}

\subsection{Compactness Estimates on Discrete Solutions}

In this subsection, we derive estimates on differences of space and time translates necessary to apply Kolmogorov's compactness theorem which will allow us to pass to the limit in the nonlinear second order terms.

Lemma 7 (Time translate estimate). There exists a constant $C>0$ depend of $\Omega$, and $T$ such that

$$
\iint_{\Omega \times[0, T-\tau]}\left(\tilde{S}_{h, \Delta t}(t+\tau, x)-\tilde{S}_{h, \Delta t}(t, x)\right)^{2} d x d t \leq C(\tau+\Delta t), \forall \tau \in[0, T] .
$$

Lemma 8 (space translate estimate). There exists a constant $C>0$ depend of $\Omega$ and $T$ such that :

$$
\left.\iint_{\Omega \times[0, T]}\left(\tilde{S}_{h, \Delta t}(t, x+\xi)\right)-\tilde{S}_{h, \Delta t}(t, x)\right)^{2} d x d t \leq C|\xi|(|\xi|+h), \forall \xi \in \mathbb{R}^{d}
$$

The estimates on the discrete global pressure (25) and on the saturation (26) are sufficient to prove the Lemma. The technics is being classical and widely used in the works of Eymard, Galouet, Herbin and their co-authors $[3,12,13,21]$.

\subsection{Convergence of the combined scheme}

This subsection is mainly devoted to the proof of the strong $L^{2}\left(Q_{T}\right)$ convergence of approximate saturation solutions, using the estimations proved in the subsection 3.2, 4.1 and Kolmogorovs compactness criterion (see [6]) for the convergence. Then, we prove that the limit is a weak solution to the continuous problem .

Lemma 9. The sequence $\left(g_{h}\left(S_{h, \Delta t}\right)-g\left(\tilde{S}_{h, \Delta t}\right)\right)_{h, \Delta t}$ converges strongly to zero in $L^{2}\left(Q_{T}\right)$ when $h \rightarrow 0$ for $g=\beta$ and $g=I d$.

Proof: Using the definition of the basis functions of the finite dimensional space $X_{h}$, and the reconstruction of discrete saturation in definition 2 give us

$$
\left|\beta\left(\tilde{S}_{h, \Delta t}\right)-\beta_{h}\left(S_{h, \Delta t}\right)\right|^{2}=\left|\beta_{h}\left(S_{h, \Delta t}\right)\left(\mathcal{P}_{D}\right)-\beta_{h}\left(S_{h, \Delta t}\right)(x)\right|^{2}=\left|\nabla \beta_{h}\left(S_{h, \Delta t}\right) \cdot\left(\mathcal{P}_{D}-x\right)\right|^{2} .
$$

Integrating over $\Omega$ leads

$$
\begin{aligned}
\sum_{K \in \mathcal{T}_{h}} \sum_{\sigma_{D} \in \mathcal{E}_{K}} \int_{K \cap D}\left|\nabla \beta_{h}\left(S_{h, \Delta t}\right) \cdot\left(\mathcal{P}_{D}-x\right)\right|^{2} d x & \leq\left.\sum_{K \in \mathcal{T}_{h}} \sum_{\sigma_{D} \in \mathcal{E}_{K}}\left|\nabla \beta_{h}\left(S_{h, \Delta t}\right)\right|_{K}\right|^{2}(\operatorname{diam}(D))^{2}|K \cap D| \\
& \leq\left. h^{2} \sum_{K \in \mathcal{T}_{h}}\left|\nabla \beta_{h}\left(S_{h, \Delta t}\right)\right|_{K}\right|^{2}|K| \leq\left. h^{2}|| \beta_{h}\left(S_{h, \Delta t}\right)\right|_{X_{h}} ^{2} .
\end{aligned}
$$

Thus, the estimate (29) gives

$$
\left\|\beta_{h}\left(S_{h, \Delta t}\right)-\beta\left(\tilde{S}_{h, \Delta t}\right)\right\|_{L^{2}\left(Q_{T}\right)} \leq h^{2} \sum_{n=0}^{N} \Delta t\left\|\beta_{h}\left(S_{h, \Delta t}\right)\right\|_{X_{h}}^{2} \leq C_{1} h^{2} \stackrel{h, \Delta t \rightarrow 0}{\longrightarrow} 0 .
$$

Same proof for $g=I d$.

Theorem 2. (Strong convergence in $\left.L^{2}\left(Q_{T}\right)\right)$ There exists a subsequence of $\left(S_{h, \Delta t}\right)_{h, \Delta t}$ which converges strongly to a function s in $L^{2}\left(Q_{T}\right)$. In addition, $\left(\beta_{h}\left(S_{h, \Delta t}\right)\right)_{h, \Delta t}$ converges strongly in $L^{2}\left(Q_{T}\right)$ to the function $\Gamma=\beta(s)$. 
Proof. Proposition 2, and lemmas 7-8 implies that the sequence $\left(\tilde{S}_{h, \Delta t}\right)_{h, \Delta t}$ satisfies the assumptions of the Kolmogorov compactness criterion, and consequently the sequence $\left(\tilde{S}_{h, \Delta t}\right)_{h, \Delta t}$ is relatively compact in $L^{2}\left(Q_{T}\right)$. Thanks to lemma 9, $\left(S_{h, \Delta t}\right)_{h, \Delta t}$ converge strongly to a function $s \in L^{2}\left(Q_{T}\right)$.

Since $\beta$ is well defined and continuous, thus, we extend it as follow :

$$
\beta(s)=\left\{\begin{array}{cl}
0, & \text { if } s \leq 0 \\
\int_{0}^{s} \alpha(z) d z, & \text { if } 0 \leq s \leq 1 \\
\int_{0}^{1} \alpha(z) d z, & \text { if } s \geq 1
\end{array}\right.
$$

applying $L^{\infty}$ bound on $\tilde{S}_{h, \Delta t}$ and the dominated convergence theorem of Lebesgue to $\beta\left(S_{h}\right)$, there exists subsequence $\beta\left(S_{h, \Delta t}\right)$ converge strongly in $L^{2}\left(Q_{T}\right)$ and a.e in $Q_{T}$ to $\beta(s)$. Finally, by Lemma 9 we deduce that $\left(\beta_{h}\left(S_{h, \Delta t}\right)\right)_{h, \Delta t}$ converges strongly in $L^{2}\left(Q_{T}\right)$ to $\Gamma=\beta(s)$.

Lemma 10. There exists a subsequence of $\left(f_{\tilde{S}_{h, \Delta t}}\right)_{h, \Delta t}$ defined in (14) which is weakly convergent in $W^{1,2}\left(0, T ; L^{2}(\Omega)\right)$ to the function s defined in theorem 2.

Proof. For the first part of convergence, it is useful to introduce the following inequality, for all $a, b \in \mathbb{R}$

$$
\int_{0}^{1}|\theta a+(1-\theta) b| d \theta \geq \frac{1}{2}(|a|+|b|)
$$

Applying this inequality to $a=\tilde{S}_{h}^{n+1}-\tilde{S}_{h}^{n}, b=\tilde{S}_{h}^{n}-\tilde{S}_{h}^{N}$, from the definition of $f_{\tilde{S}_{h, \Delta t}}$ we deduce

$$
\int_{0}^{T} \int_{\Omega}\left|f_{\tilde{S}_{h, \Delta t}}(t, x)-\tilde{S}_{h, \Delta t}(t, x)\right| d x d t \leq \int_{0}^{T-\Delta t} \int_{\Omega}\left|\tilde{S}_{h, \Delta t}(t+\Delta t, x)-\tilde{S}_{h, \Delta t}(t, x)\right| d x d t .
$$

Since $\Delta t$ tends to zero, the translate in time estimate in Lemma 7 implies that the right-hand side of the above inequality converges to zero as $h$ and $\Delta t$ tends to zero. Therefore, since we obtain a strongly convergence in $L^{1}\left(Q_{T}\right)$ and by construction of $f_{S}$ we have that $f_{\tilde{S}_{h, \Delta t}}$ is bounded in $L^{2}\left(Q_{T}\right)$, this yield to deduce that $f_{\tilde{S}_{h, \Delta t}}$ weakly converge to $s$ in $L^{2}\left(0, T ; L^{2}(\Omega)\right)$. Next, for $\psi \in \Theta=\left\{u \in C^{1,1}([0, T] \times \bar{\Omega}), u(T,)=0.\right\}$, we denote by $\psi_{D}^{n}=\psi\left(t_{n}, x_{D}\right)$ and we define

$$
\begin{aligned}
T_{T} & =-\sum_{n=0}^{N} \sum_{D \in \mathcal{D}_{h}}|D| S_{D}^{n+1}\left(\psi_{D}^{n}-\psi_{D}^{n+1}\right)-\sum_{D \in \mathcal{D}_{h}^{i n t}}|D| S_{D}^{0}\left(\psi_{D}^{0}\right) \\
& =-\sum_{n=0}^{N} \sum_{D \in \mathcal{D}_{h}} \int_{t_{n}}^{t_{n+1}} \int_{D} S_{D}^{n+1} \frac{\partial \psi}{\partial t}\left(t, P_{D}\right) d x d t-\sum_{D \in \mathcal{D}_{h}} \int_{D} S_{D}^{0} \psi\left(0, x_{D}\right) d x
\end{aligned}
$$

also we define

$$
\begin{aligned}
\tilde{T}_{T} & =-\iint_{Q_{T}} \tilde{S}_{h, \Delta t} \frac{\partial \psi}{\partial t} d x d t-\int_{\Omega} \tilde{S}_{0, h}(0, x) \psi(x, 0) d x \\
& =-\sum_{n=0}^{N} \sum_{D \in \mathcal{D}_{h}} \int_{t_{n}}^{t_{n+1}} \int_{D} S_{D}^{n+1} \frac{\partial \psi}{\partial t}(t, x) d x d t-\sum_{D \in \mathcal{D}_{h}} \int_{D} S_{D}^{0} \psi(0, x) d x
\end{aligned}
$$

We have, for all $x \in D, D \in \mathcal{D}_{h}$ and for all $h>0,\left|\frac{\partial \psi}{\partial t}\left(t, P_{D}\right)-\frac{\partial \psi}{\partial t}(t, x)\right| \leq \varepsilon(h)$, where the function $\varepsilon$ satisfies $\varepsilon(h)>0$ and $\varepsilon(h) \stackrel{h \rightarrow 0}{\longrightarrow} 0$. This follows by the fact that $\frac{\partial \psi}{\partial t} \in C^{0}(\bar{\Omega})$, then $\frac{\partial \psi}{\partial t}$ is uniformly continuous on $\bar{\Omega}$. Consequently, there exist a constant $C>0$ such that

$$
\left|T_{T}-\tilde{T}_{T}\right| \leq C \varepsilon(h) \stackrel{h, \Delta t \rightarrow 0}{\longrightarrow} 0 .
$$


FV scheme for Darcy-Brinkman's model of two-phase flows

Note that $\tilde{S}_{h, \Delta t}$ converge also to s strongly in $L^{2}\left(Q_{T}\right)$, then we have but by theorem 2 and Lemma 9 we have,

$$
\tilde{T}_{T} \rightarrow-\iint_{Q_{T}} s \frac{\partial \psi}{\partial t} d x d t-\int_{\Omega} s(0, x) \psi(x, 0) d x
$$

Notice that,

$$
T_{T}=\sum_{n=0}^{N} \sum_{D \in \mathcal{D}_{h}}|D| \frac{S_{D}^{n+1}-S_{D}^{n}}{\Delta t} \Delta t \psi_{D}^{n+1}=\iint_{Q_{T}} \frac{\partial f_{\tilde{S}_{h, \Delta t}}}{\partial t} \psi_{h} \rightarrow-\iint_{Q_{T}} s \frac{\partial \psi}{\partial t} d x d t-\int_{\Omega} s(0, x) \psi(0, x) d x
$$

From estimate (28), the sequence $\left(f_{\tilde{S}}\right)_{h, \Delta t}$ is bounded in $L^{2}\left(Q_{T}\right)$, then there a exist a function $\zeta$ such that $\left(f_{\tilde{S}}\right)_{h, \Delta t}$ converge to $\zeta$ weakly in $L^{2}\left(Q_{T}\right)$, thus

$$
\iint_{Q_{T}} \zeta \psi d t d x=-\iint_{Q_{T}} s \frac{\partial \psi}{\partial t} d x d t-\int_{\Omega} s(0, x) \psi(0, x) d x=\iint_{Q_{T}} \frac{\partial f_{\tilde{S}_{h, \Delta t}}}{\partial t} \psi d t d x, \quad \forall \psi \in \Theta,
$$

therefor, we can deduce that $\zeta=\frac{\partial f_{\tilde{S}_{h, \Delta t}}}{\partial t}$ and $\iint_{Q_{T}} \frac{\partial f_{\tilde{S}_{h, \Delta t}}}{\partial t} \psi \rightarrow \iint_{Q_{T}} \frac{\partial s}{\partial t} \psi, \quad \forall \psi \in \Theta$. Furthermore, if we take $\psi=\phi-\phi(T,$.$) for all \phi \in C^{1,1}([0, T] \times \bar{\Omega})$, we obtain

$$
\iint_{Q_{T}} \frac{\partial f_{\tilde{S}_{h, \Delta t}}}{\partial t} \phi-\iint_{Q_{T}} \frac{\partial f_{\tilde{S}_{h, \Delta t}}}{\partial t} \phi(T, .) \rightarrow \iint_{Q_{T}} \frac{\partial s}{\partial t} \phi-\int_{Q_{T}} \frac{\partial s}{\partial t} \phi(T, .) \quad \forall \phi \in C^{1,1}([0, T] \times \bar{\Omega}) .
$$

then, it is enough to show

$$
\iint_{Q_{T}} \frac{\partial f_{\tilde{S}_{h, \Delta t}}}{\partial t} \phi(T, .) \rightarrow \int_{Q_{T}} \frac{\partial s}{\partial t} \phi(T, .) \quad \forall \phi \in C^{1,1}([0, T] \times \bar{\Omega}),
$$

to proof that

$$
\iint_{Q_{T}} \frac{\partial f_{\tilde{S}_{h, \Delta t}}}{\partial t} \phi \rightarrow \iint_{Q_{T}} \frac{\partial s}{\partial t} \phi \quad \forall \phi \in C^{1,1}([0, T] \times \bar{\Omega}) .
$$

An integration by parts with respect to $t$ gives us

$\iint_{Q_{T}} \frac{\partial f_{\tilde{S}_{h, \Delta t}}}{\partial t} \phi(T, x) d x d t=\int_{\Omega} f_{\tilde{S}_{h, \Delta t}}(T, x) \phi(T, x) d x-\int_{\Omega} f_{\tilde{S}_{h, \Delta t}}(0, x) \phi(T, x) d x=\int_{\Omega} \tilde{S}_{h}^{N+1} \phi(T, x) d x-\int_{\Omega} \tilde{S}_{h}^{0} \phi(T, x) d x$,

by proposition 2 and by applying Lemma 8 for $\tilde{S}_{h}^{N+1}, \tilde{S}_{h}^{0}$ respectively, we deduce by Kolmogorov's theorem that $\tilde{S}_{h}^{N+1}, \tilde{S}_{h}^{0} \rightarrow s(T,),. s(0,$.$) , strongly in L^{2}(\Omega)$. Then

$$
\iint_{Q_{T}} \frac{\partial f_{\tilde{S}_{h, \Delta t}}}{\partial t} \phi(T, .) d x d t \rightarrow \int_{\Omega} s(T, .) \phi(T, .)-\int_{\Omega} s(0, .) \phi(T, .)=\int_{Q_{T}} \frac{\partial s}{\partial t} \phi(T, .), \forall \phi \in C^{1,1}([0, T] \times \bar{\Omega}),
$$

Finally, by using the density of $C^{1,1}([0, T] \times \bar{\Omega})$ in $L^{2}\left(Q_{T}\right)$ we obtain

$$
\iint_{Q_{T}} \frac{\partial f_{\tilde{S}_{h, \Delta t}}}{\partial t} \phi \rightarrow \iint_{Q_{T}} \frac{\partial s}{\partial t} \psi \quad \forall \phi \in L^{2}\left(Q_{T}\right) .
$$




\section{Proof of theorem 1}

We will prove now that the limit couple $(s, p)$ is a weak solution of the continuous problem. We take $\psi \in$ $C^{1,1}([0, T] \times \bar{\Omega})$. We then multiply (7) by $\Delta t \psi\left(t_{n+1}, \mathcal{P}_{D}\right)$ and we sum the result over $D \in \mathcal{D}_{h}^{\text {int }}$ and for all $n \in[0, \ldots, N]$, we have :

$$
\mathcal{E}_{1}+\mathcal{E}_{2}+\mathcal{E}_{3}+\mathcal{E}_{4}=\mathcal{E}_{5},
$$

with,

$$
\begin{aligned}
& \mathcal{E}_{1}=\sum_{n=0}^{N} \Delta t \sum_{D \in \mathcal{D}_{h}}|D| \frac{S_{D}^{n+1}-S_{D}^{n}}{\Delta t} \psi\left(t_{n+1}, \mathcal{P}_{D}\right), \\
& \mathcal{E}_{2}=-\mu \sum_{n=0}^{N} \Delta t \sum_{D \in \mathcal{D}_{h}} \sum_{E \in \mathcal{N}(D)} \mathcal{N}_{D, E}\left(\frac{S_{E}^{n+1}-S_{E}^{n}}{\Delta t}-\frac{S_{D}^{n+1}-S_{D}^{n}}{\Delta t}\right) \psi\left(t_{n+1}, \mathcal{P}_{D}\right), \\
& \mathcal{E}_{3}=-\sum_{n=0}^{N} \Delta t \sum_{D \in \mathcal{D}_{h}} \sum_{E \in \mathcal{N}(D)} \mathcal{M}_{D, E}\left(\beta\left(S_{E}^{n+1}\right)-\beta\left(S_{D}^{n+1}\right)\right) \psi\left(t_{n+1}, \mathcal{P}_{D}\right), \\
& \mathcal{E}_{4}=\sum_{n=0}^{N} \Delta t \sum_{D \in \mathcal{D}_{h}} \sum_{E \in \mathcal{N}(D)} G_{s}\left(S_{D}^{n+1}, S_{E}^{n+1} ; \delta P_{D, E}^{n+1}\right) \psi\left(t_{n+1}, \mathcal{P}_{D}\right), \\
& \mathcal{E}_{5}=\sum_{n=0}^{N} \sum_{D \in \mathcal{D}_{h}} \Delta t|\partial D \cap \partial \Omega| h_{D}^{n+1} \psi\left(t_{n+1}, \mathcal{P}_{D}\right) .
\end{aligned}
$$

We now show that each of the above terms converges to its continuous version as $h, \Delta t \rightarrow 0$. The first term is equal to

$$
\mathcal{E}_{1}=\sum_{n=0}^{N} \sum_{D \in \mathcal{D}_{h}} \int_{t_{n}}^{t_{n+1}} \int_{D} \frac{\partial f_{\tilde{S}_{h}}}{\partial t} \psi\left(t_{n+1}, \mathcal{P}_{D}\right) d x d t
$$

Define

$$
\tilde{\mathcal{E}}_{1}=\iint_{Q_{T}} \frac{\partial f_{\tilde{S}_{h}}}{\partial t} \psi d x d t=\sum_{n=0}^{N} \sum_{D \in \mathcal{D}_{h}} \int_{t_{n}}^{t_{n+1}} \int_{D} \frac{\partial f_{\tilde{S}_{h}}}{\partial t} \psi(t, x) d x d t .
$$

We have, for all $x \in D, D \in \mathcal{D}_{h}$ and for all $h>0, \Delta t>0,\left|\psi\left(t_{n+1}, \mathcal{P}_{D}\right)-\psi(t, x)\right| \leq \varepsilon(h, \Delta t)$, as we have seen before, so we have

$$
\left|\mathcal{E}_{1}-\tilde{\mathcal{E}}_{1}\right| \leq C \varepsilon(h, \Delta t) \stackrel{h, \Delta t \rightarrow 0}{\longrightarrow} 0 .
$$

Lemma 10 ensures that $\frac{\partial f_{\tilde{S}_{h}}}{\partial t} \rightarrow \partial_{t} s$ weakly in $L^{2}\left(Q_{T}\right)$, then

$$
\mathcal{E}_{1} \longrightarrow \iint_{Q_{T}} \partial_{t} s \psi d x d t
$$

According to Remark 2, we can rewrite $\mathcal{E}_{2}$ as follow 
FV scheme for Darcy-Brinkman's model of two-phase flows

$$
\begin{aligned}
\mathcal{E}_{2} & =-\mu \sum_{n=0}^{N} \Delta t \sum_{D \in \mathcal{D}_{h}} \sum_{E \in \mathcal{D}_{h}} \mathcal{N}_{D, E}\left(\frac{S_{E}^{n+1}-S_{E}^{n}}{\Delta t}\right) \psi\left(t_{n+1}, \mathcal{P}_{D}\right) \\
& =\mu \sum_{n=0}^{N} \Delta t \sum_{K \in \mathcal{T}_{h}} \int_{K} \nabla \partial_{t} f_{S_{h}} \cdot \nabla\left(\sum_{D \in \mathcal{D}_{h}} \psi\left(t_{n+1}, \mathcal{P}_{D}\right) \varphi_{D}(x)\right) d x .
\end{aligned}
$$

We set

$$
I_{\psi}\left(t_{n+1}, x\right):=\sum_{D \in \mathcal{D}_{h}} \psi\left(t_{n+1}, \mathcal{P}_{D}\right) \varphi_{D}(x)
$$

we will prove that

$$
\mathcal{E}_{2,1}=\mu \sum_{n=0}^{N} \Delta t \sum_{K \in \mathcal{T}_{h}} \int_{K} \nabla \partial_{t} f_{S_{h}} \cdot \nabla\left(I_{\psi}\left(t_{n+1}, x\right)-\psi\left(t_{n+1}, x\right)\right) d x \stackrel{h \rightarrow 0}{\longrightarrow} 0 .
$$

Indeed, using Cauchy-Schwarz inequality, we estimate :

$$
\begin{aligned}
\left|\mathcal{E}_{2,1}\right| & \leq \mu c_{I} \sum_{n=0}^{N} \Delta t|| \partial_{t} f_{S_{h}^{n+1}}||_{X_{h}}\left(\sum_{K \in \mathcal{T}_{h}} \int_{K}\left|\nabla\left(I_{\psi}\left(t_{n+1}, x\right)-\psi\left(t_{n+1}, x\right)\right)\right|^{2} d x\right)^{\frac{1}{2}} \\
& \leq \mu c_{I} \sum_{n=0}^{N} \Delta t|| \partial_{t} f_{S_{h}^{n+1}}||_{X_{h}}\left(C \theta_{\mathcal{T}} h\left(\sum_{K \in \mathcal{T}_{h}}\left|\psi\left(t_{n+1}, x\right)\right|_{2, K}^{2}\right)^{\frac{1}{2}}\right),
\end{aligned}
$$

where $|\cdot|_{2, K}$ denotes the $H^{2}$ seminorm and the constant $C$ does not depend on $h$ (nor on $\Delta t$ ) see for instance [10, Theorem 15.3]. Finally, using Cauchy-Schwarz inequality, we conclude that

$$
\left|\mathcal{E}_{2,1}\right| \leq \mu c_{I} C \theta_{\mathcal{T}} h C_{\psi}\left(\sum_{n=0}^{N}\left\|\partial_{t} f_{S_{h}^{n+1}}\right\|_{X_{h}}^{2}\right)^{\frac{1}{2}}\left(\sum_{n=0}^{N} \Delta t\right)^{\frac{1}{2}} \stackrel{h, \Delta t \rightarrow 0}{\longrightarrow} 0 .
$$

Then, we can proof that

$$
\mathcal{E}_{2} \longrightarrow \mu \iint_{Q_{T}} \nabla \partial_{t} s \cdot \nabla \psi d x d t .
$$

For more details see the convergence of diffusion term in what follow.

To study the convergence of diffusion term, we use the same technic as in [24]. At first, according to remark 2 and if we replace $M_{D, E}$ by its value, we obtain :

$$
\mathcal{E}_{3}=\sum_{n=0}^{N} \sum_{K \in \mathcal{T}_{h}} \int_{K} \Lambda \nabla \beta_{h}\left(S_{h}^{n+1}(x)\right) \cdot \nabla\left(\sum_{D \in \mathcal{D}_{h}} \psi\left(t_{n+1}, \mathcal{P}_{D}\right) \varphi_{D}(x)\right) d x .
$$

a) As a first step, we use the boundedness of the tensor $\Lambda$ and same technic as before to prove that

$$
\mathcal{E}_{3,1}=\sum_{n=0}^{N} \sum_{K \in \mathcal{T}_{h}} \int_{K} \Lambda \nabla \beta_{h}\left(S_{h}^{n+1}(x)\right) \cdot \nabla\left(I_{\psi}\left(t_{n+1}, x\right)-\psi\left(t_{n+1}, x\right)\right) d x \stackrel{h, \Delta t \rightarrow 0}{\longrightarrow} 0 .
$$

b) We next show that:

$$
\sum_{n=0}^{N} \Delta t \sum_{K \in \mathcal{T}_{h}} \int_{K} \Lambda \nabla \beta_{h}\left(S_{h}^{n+1}(x)\right) \cdot \nabla \psi\left(t_{n+1}, x\right) d x d t-\iint_{Q_{T}} \Lambda \nabla \beta(s) \cdot \nabla \psi d x d t \stackrel{h, \Delta t \rightarrow 0}{\longrightarrow} 0 .
$$


For that, we add and subtract $\int_{Q_{T}} \Lambda \nabla \beta_{h}\left(S_{h}^{n+1}\right) \cdot \nabla \psi d x d t$ and consider

$$
\begin{aligned}
& \mathcal{E}_{3,2}=\sum_{n=0}^{N} \int_{t_{n}}^{t_{n+1}} \sum_{K \in \mathcal{T}_{h}} \int_{K} \Lambda \nabla \beta_{h}\left(S_{h}^{n+1}(x)\right) \cdot \nabla\left(\psi\left(t_{n+1}, x\right)-\psi(x, t)\right) d x d t \\
& \mathcal{E}_{3,3}=\iint_{Q_{T}} \Lambda\left(\nabla \beta_{h}\left(S_{h}(x)\right)-\nabla \beta(s)\right) \cdot \nabla \psi(x, t) d x d t .
\end{aligned}
$$

Since $\psi$ is of class $C^{1}$ with respect to $t$, then $\left|\nabla \psi\left(t_{n+1}, x\right)-\nabla \psi(x, t)\right| \leq g(\Delta t) \stackrel{\Delta t \rightarrow 0}{\longrightarrow} 0$ and

$$
\left|\mathcal{E}_{3,2}\right| \leq C_{\Lambda} g(\Delta t) \sum_{n=0}^{N} \sum_{K \in \mathcal{T}_{h}}\left|\nabla \beta_{h}\left(S_{h}^{n+1}\right)_{\left.\right|_{K} \mid}\right| K \mid .
$$

The Cauchy-Schwarz inequality and the estimate (29) give

$$
\left|\mathcal{E}_{3,2}\right| \leq C_{\Lambda} g(\Delta t) T^{\frac{1}{2}}\left(\left.\sum_{n=0}^{N} \Delta t|| \beta_{h}\left(S_{h}^{n+1}\right)\right|_{X_{h}} ^{2}\right)^{\frac{1}{2}} \leq C C_{\Lambda} g(\Delta t) T^{\frac{1}{2}}|\Omega|^{\frac{1}{2}} \stackrel{\Delta t \rightarrow 0}{\longrightarrow} 0 .
$$

Now, we still have to prove that

$$
\mathcal{E}_{3,3}^{\prime}=\iint_{Q_{T}}\left(\nabla \beta_{h}\left(S_{h}(x)\right)-\nabla \beta(s)\right) \cdot w(x, t) d x d t \stackrel{h, \Delta t \rightarrow 0}{\longrightarrow} 0,
$$

for all $w \in\left(C^{\infty}\left(Q_{T}\right)\right)^{d}$. Indeed,

$$
\begin{aligned}
\mathcal{E}_{3,3}^{\prime} & =\iint_{Q_{T}} \nabla \beta_{h}\left(S_{h}(x)\right) \cdot w(x, t) d x d t+\iint_{Q_{T}} \beta(s(x, t)) \nabla \cdot w(x, t) d x d t-\int_{0}^{T} \int_{\partial \Omega} \beta(s(x, t)) w(x, t) \cdot \eta d \gamma(x) d t \\
& =B_{1}+B_{2}+B_{3}
\end{aligned}
$$

where we use the Green formula in $B_{2}$ because $\beta(s) \in L^{2}\left(0, T ; H^{1}(\Omega)\right)$ but in $B_{1}$ we apply the Green formula for each $K \in \mathcal{T}_{h}$ because $\beta_{h}\left(S_{h}^{n+1}\right) \notin H^{1}(\Omega)$. For that,

$$
B_{1}=-\int_{0}^{T} \sum_{K \in \mathcal{T}_{h}} \int_{K} \beta_{h}\left(S_{h}(x)\right) \nabla \cdot(w(x, t)) d x d t+\int_{0}^{T} \sum_{K \in \mathcal{T}_{h}} \int_{\partial K} \beta_{h}\left(S_{h}(x)\right) w(x, t) \cdot \eta d \gamma(x) d t
$$

By reordering the summation of the second term by sides, we have

$$
\begin{aligned}
B_{1}^{\prime}=\int_{0}^{T} \sum_{\sigma_{K, L} \in \mathcal{E}_{h}^{\text {int }}} \int_{\sigma_{K, L}}\left(\left.\beta_{h}\left(S_{h}(x)\right)\right|_{K}-\left.\beta_{h}\left(S_{h}(x)\right)\right|_{L}\right) w(x, t) \cdot \eta d \gamma(x) d t & \\
& +\int_{0}^{T} \sum_{\sigma_{K} \in \mathcal{E}_{h}^{\text {ext }}} \int_{\sigma_{K}}\left(\left.\beta_{h}\left(S_{h}(x)\right)\right|_{K}\right) w(x, t) \cdot \eta d \gamma(x) d t .
\end{aligned}
$$

Since the function $w$ is smooth, we get

$$
w \cdot \eta_{\sigma_{D}}(x)=w \cdot \eta_{\sigma_{D}}\left(\mathcal{P}_{D}\right)+f(\xi)\left|\mathcal{P}_{D}-x\right|, x \in \sigma_{D}, \xi \in\left[\mathcal{P}_{D}, x\right],
$$

with $|f(\xi)| \leq C_{w}$. Thus, 
FV scheme for Darcy-Brinkman's model of two-phase flows

$$
\begin{aligned}
B_{1}^{\prime}=\int_{0}^{T} \sum_{\sigma_{K, L} \in \mathcal{E}_{h}^{\text {ext }}} \int_{\sigma_{K, L}}\left(\left.\beta_{h}\left(S_{h}(x)\right)\right|_{K}-\left.\beta_{h}\left(S_{h}(x)\right)\right|_{L}\right)\left(w \cdot \eta_{\sigma_{D}}\left(\mathcal{P}_{D}\right)\right. & \left.+f(\xi)\left|\mathcal{P}_{D}-x\right|\right) d \gamma(x) d t \\
& +\int_{0}^{T} \int_{\partial \Omega} \beta_{h}\left(S_{h}(x)\right) w(x, t) \cdot \eta d \gamma(x) d t
\end{aligned}
$$

The function $\left.\beta_{h}\left(S_{h}^{n+1}(x)\right)\right|_{K}-\left.\beta_{h}\left(S_{h}^{n+1}(x)\right)\right|_{L}$ is a first-order polynomial, vanishing at the barycenter $\mathcal{P}_{D}$ of this side. Consequently

$$
\int_{\sigma_{K, L}}\left(\left.\beta_{h}\left(S_{h}^{n+1}(x)\right)\right|_{K}-\left.\beta_{h}\left(S_{h}^{n+1}(x)\right)\right|_{L}\right) d \gamma(x)=0 .
$$

In addition, we have for all $x \in \sigma_{D}=\sigma_{K, L}$,

$$
\left|\beta_{h}\left(S_{h}^{n+1}(x)\right)\right|_{K}-\left.\left.\beta_{h}\left(S_{h}^{n+1}(x)\right)_{L}|\leq| \nabla \beta_{h}\left(S_{h}^{n+1}(x)\right)\right|_{K}\left|\frac{\operatorname{diam}\left(\sigma_{K, L}\right)}{4-d}+\right| \nabla \beta_{h}\left(S_{h}^{n+1}(x)\right)\right|_{L} \mid \frac{\operatorname{diam}\left(\sigma_{K, L}\right)}{4-d}
$$

here we use that $\left|x-\mathcal{P}_{D}\right| \leq \operatorname{diam}\left(\sigma_{K, L}\right) / 2$ for $d=2$ and $\left|x-\mathcal{P}_{D}\right| \leq \operatorname{diam}\left(\sigma_{K, L}\right)$ for $d=3$. Thus, there exists a constant $C$ depend on $C_{f}$ and $d$ such that

$$
\begin{aligned}
& \sum_{\sigma_{K, L} \in \mathcal{E}_{h}^{\text {int }}} \int_{\sigma_{K, L}}\left(\left.\beta_{h}\left(S_{h}^{n+1}(x)\right)\right|_{K}\right.\left.-\left.\beta_{h}\left(S_{h}^{n+1}(x)\right)\right|_{L}\right) f(\xi)\left|\mathcal{P}_{D}-x\right| d \gamma(x) \\
& \leq C \sum_{\sigma_{K, L} \in \mathcal{E}_{h}^{\text {int }}}\left|\sigma_{K, L}\right|\left(\left|\nabla \beta_{h}\left(S_{h}^{n+1}(x)\right)\right|_{K}|+| \nabla \beta_{h}\left(S_{h}^{n+1}(x)\right)\left|{ }_{L}\right|\right)\left(\frac{\operatorname{diam}\left(\sigma_{K, L}\right)}{4-d}\right)^{2} .
\end{aligned}
$$

Finally, it's remains to show that

$$
\int_{0}^{T} \int_{\partial \Omega} \beta_{h}\left(S_{h}(x)\right) w(x, t) \cdot \eta d \gamma(x) d t-\int_{0}^{T} \int_{\partial \Omega} \beta(s(x, t)) w(x, t) \cdot \eta d \gamma(x) d t \rightarrow 0,
$$

By applying Lemma 3 on $\beta_{h}$ and by using Theorem 2 we have the result. By reordering all estimates and using Cauchy-schwarz inequality, we can easily deduce that $B_{1}^{\prime}$ tends to $\int_{0}^{T} \int_{\partial \Omega} \beta(s(x, t)) w(x, t) \cdot \eta d \gamma(x)$ where $h$ tends to zero.

According to the convergence of $\beta_{h}\left(S_{h}^{n+1}\right)$ to $\beta(s)$ in $L^{2}\left(Q_{T}\right)$, we obtain that $\mathcal{E}_{3,3}^{\prime}$ tend to 0 for all $w \in\left(C^{\infty}\left(Q_{T}\right)\right)^{d}$, consequently $\mathcal{E}_{3,3}^{\prime}$ tends to 0 for all $w \in L^{2}\left(Q_{T}\right)$ by using the density argument.

Since $\mathcal{E}_{3,2}$ and $\mathcal{E}_{3,3}$ tends to 0 , then, the convergence of a step $b$ ) is proved. By reordering the convergences of steps $a$ ) and $b$ ), we deduce:

$$
\mathcal{E}_{3} \stackrel{h, \Delta t \rightarrow 0}{\longrightarrow} \iint_{Q_{T}} \Lambda \nabla \beta(s) \cdot \nabla \psi d x d t
$$

we are concerned now with the convective term:

$$
\mathcal{E}_{4}=\sum_{n=0}^{N} \sum_{D \in \mathcal{D}_{h}} \sum_{E \in \mathcal{N}(D)} G_{s}\left(S_{D}^{n+1}, S_{E}^{n+1} ; \delta P_{D, E}^{n+1}\right) \psi_{D}^{n+1}
$$

For each couple of neighbor volumes $D$ and $E$ we introduce

$$
S_{D, E}^{n+1}=\min \left(S_{D}^{n+1}, S_{E}^{n+1}\right),
$$

and we set 


$$
\mathcal{E}_{4}^{*}=-\sum_{n=0}^{N} \Delta t \sum_{D \in \mathcal{D}_{h}} \sum_{E \in \mathcal{N}(D)} f\left(S_{D, E}^{n+1}\right) \lambda\left(S_{D, E}^{n+1}\right) \delta P_{D, E}^{n+1} \psi_{D}^{n+1} .
$$

The diamond constructed from the neighbor edge centers $\mathcal{P}_{D}, \mathcal{P}_{E}$ and the interface $\sigma_{D, E}$ of the dual mesh is denoted by $T_{D, E} \subset K_{D, E}$. Then, we introduce

$$
\left.\overline{S_{h}}\right|_{\left.\mid t_{n}, t_{n+1}\right] \times T_{D, E}}:=\max \left(S_{D}^{n+1}, S_{E}^{n+1}\right),\left.\quad \underline{S_{h}}\right|_{\left.\mid t_{n}, t_{n+1}\right] \times T_{D, E}}:=\min \left(S_{D}^{n+1}, S_{E}^{n+1}\right),
$$

and we rewrite

$$
\mathcal{E}_{4}^{*}=\iint_{Q_{T}} \Lambda f\left(\underline{S_{h}}\right) \lambda\left(\underline{S_{h}}\right) \nabla P_{h} \cdot(\nabla \psi)_{h} d x d t .
$$

By the monotonicity of $\beta$ and thanks to the estimate (16), we have

$$
\begin{aligned}
\int_{0}^{T} \int_{\Omega}\left|\beta\left(\overline{S_{h}}\right)-\beta\left(\underline{S_{h}}\right)\right|^{2} & \leq \sum_{n=0}^{N} \Delta t \sum_{D \in \mathcal{D}_{h}} \sum_{E \in \mathcal{N}(D)}\left|T_{D, E}\right|\left|\beta\left(S_{D}^{n+1}\right)-\beta\left(S_{E}^{n+1}\right)\right|^{2} \\
& \leq \sum_{n=0}^{N} \Delta t \sum_{D \in \mathcal{D}_{h}} \sum_{E \in \mathcal{N}(D)}\left(\operatorname{diam}\left(K_{D, E}\right)\right)^{2}\left|\beta\left(S_{D}^{n+1}\right)-\beta\left(S_{E}^{n+1}\right)\right|^{2} \\
& \leq C h^{4-d} \stackrel{h, \Delta t \rightarrow 0}{\longrightarrow} 0,
\end{aligned}
$$

with $d=2$ or 3 . Because $\beta^{-1}$ is continuous, up to extraction of another subsequence, we deduce

$$
\left|\overline{S_{h}}-\underline{S_{h}}\right| \stackrel{h, \Delta t \rightarrow 0}{\longrightarrow} 0 \text { p.p. sur } Q_{T} .
$$

In addition, $\underline{S_{h}} \leq \tilde{S}_{h, \Delta t} \leq \overline{S_{h}}$ and $\tilde{S}_{h, \Delta t} \stackrel{h, \Delta t \rightarrow 0}{\longrightarrow} s$ a.e on $Q_{T}$. Thus, we see that $f\left(\underline{S_{h}}\right) \lambda\left(\underline{S_{h}}\right) \stackrel{h, \Delta t \rightarrow 0}{\longrightarrow} f(s) \lambda(s)$ a.e on $Q_{T}$. The dominated convergence theorem of Lebesgue implies that $\Lambda f\left(\underline{S_{h}}\right) \lambda\left(\underline{S_{h}}\right)(\nabla \psi)_{h} \longrightarrow \Lambda f(s) \lambda(s) \nabla \psi$ dans $\left(L^{2}\left(Q_{T}\right)\right)^{d}$. Using the weak convergence of the gradient of the discrete solution $P_{h}$ in $\left(L^{2}\left(Q_{T}\right)\right)^{d}$ (treated as for $\beta_{h}\left(S_{h}\right)$ in (31)), we can deduce that

$$
\mathcal{E}_{4}^{*} \stackrel{h, \Delta t \rightarrow 0}{\longrightarrow} \iint_{Q_{T}} \Lambda f(s) \lambda(s) \nabla p \cdot \nabla \psi d x d t
$$

It remains now to show that

$$
\lim _{h \rightarrow 0}\left|\mathcal{E}_{4}-\mathcal{E}_{4}^{*}\right|=0
$$

By properties of $G_{s}$, we have

$$
\begin{aligned}
\mid G_{s}\left(S_{D}^{n+1}, S_{E}^{n+1}, \delta P_{D, E}^{n+1}\right)- & f\left(S_{D, E}^{n+1}\right) \lambda\left(S_{D, E}^{n+1}\right) \delta P_{D, E}^{n+1} \mid \\
& =\left|G_{s}\left(S_{D}^{n+1}, S_{E}^{n+1}, \delta P_{D, E}^{n+1}\right)-\right| G_{s}\left(S_{D, E}^{n+1}, S_{D, E}^{n+1}, \delta P_{D, E}^{n+1}\right) \mid \\
& \leq C\left|\delta P_{D, E}^{n+1}\right|\left|\overline{S_{h}}-\underline{S_{h}}\right|_{D, E} .
\end{aligned}
$$

Therefore,

$$
\left|\mathcal{E}_{4}-\mathcal{E}_{4}^{*}\right| \leq \frac{C}{2} \sum_{n=0}^{N} \Delta t \sum_{D \in \mathcal{D}_{h}} \sum_{E \in \mathcal{N}(D)} \mathcal{M}_{D, E}\left|P_{E}^{n+1}-P_{D}^{n+1}\right|\left|\overline{S_{h}}-\underline{S_{h}}\right| T_{D, E}\left|\psi_{E}^{n+1}-\psi_{D}^{n+1}\right|
$$

Using Youngs inequality, the estimate (25) and the Taylor formula for $\psi$, one can easily deduce (33). Similarly, for the last term $\mathcal{E}_{5}$ we have 


$$
\mathcal{E}_{5}=\sum_{n=0}^{N} \sum_{D \in \mathcal{D}_{h}}|\partial D \cap \partial \Omega| h_{D}^{n+1} \psi\left(t_{n+1}, \mathcal{P}_{D}\right)=\sum_{n=0}^{N} \sum_{D \in \mathcal{D}_{h}} \int_{t_{n}}^{t_{n+1}} \int_{D} h(t, x) \psi\left(t_{n+1}, \mathcal{P}_{D}\right),
$$

by continuity of $\psi$ we have

$$
\mathcal{E}_{5} \stackrel{h, \Delta t \rightarrow 0}{\longrightarrow} \int_{0}^{T} \int_{\partial \Omega} h \psi d x d t
$$

Otherwise, for the second equation of scheme (7)-(9) we use the same proof ( as for convective term ) to obtain

$$
\int_{0}^{T} \int_{\Omega} \lambda(s) \Lambda \nabla p \cdot \nabla \varphi d x d t=\int_{0}^{T} \int_{\partial \Omega} \pi \varphi d x d t \forall \psi \in C^{1,1}([0, T] \times \bar{\Omega}) .
$$

Finally,we conclude that the limit couple $(s, p)$ is a weak solution of the continuous problem in the sense of definition 1 by using the fact that the space $C^{1,1}([0 . T] \times \bar{\Omega})$ is dense in $L^{2}\left(0, T ; H^{1}(\Omega)\right)$.

\section{References}

1. Angot, P. Dolejsi, V. Feistauer, M., and Felcman, J. : Analysis of a combined barycentric finite volume-nonconforming finite element method for nonlinear convection-diffusion problems. Applications of Mathematics 43.4 (1998): 263-310.

2. Auriault, J. L., Geindreau, C., and Boutin, C. : Filtration law in porous media with poor separation of scales. Transport in porous media 60.1 (2005): 89-108.

3. Bendahmane, M., Khalil, Z., and Saad, M. : Convergence of a finite volume scheme for gas water flow in a multi-dimensional porous medium. Mathematical Models and Methods in Applied Sciences 24.01 (2014): 145-185.

4. Brenner, S. and Ridgway S. : The mathematical theory of finite element methods. Vol. 15. Springer Science and Business Media, 2007.

5. Brenner, K., Cancès, C., and Hilhorst, D. : Finite volume approximation for an immiscible two-phase flow in porous media with discontinuous capillary pressure. Computational Geosciences 17.3 (2013): 573-597.

6. Brezis, H. : Analyse fonctionnelle: Théorie et applications. Vol. 91. Paris: Dunod, 1999.

7. Brinkman, H. C. : A calculation of the viscous force exerted by a flowing fluid on a dense swarm of particles. Applied Scientific Research 1.1 (1949): 27-34.

8. Chavent, G., and Jaffré, J. : Mathematical models and finite elements for reservoir simulation: single phase, multiphase and multicomponent flows through porous media. Vol. 17. Elsevier, 1986.

9. Chen, Z., Ewing, R. E., and Espedal, M. : Multiphase flow simulation with various boundary conditions. Computational Methods in Water Resources (1994): 925-932.

10. Ciarlet, P. G. : Basic error estimates for elliptic problems, Handbook of numerical analysis,Vol. 2, Elsevier Science B.V., Amesterdam, 1991, pp. 17-351.

11. Coclite, G. M., Mishra, S., Risebro, N. H., and Weber, F. : Analysis and numerical approximation of Brinkman regularization of two-phase flows in porous media. Computational Geosciences 18.5 (2014): 637-659.

12. Coudière, Y., Saad, M. and Uzureau, A. : Analysis of a finite volume method for a bone growth system in vivo. Computers and Mathematics with Applications 66.9 (2013): 1581-1594.

13. Eymard, R., Danielle H., and Vohralk, M. : A combined finite volume-nonconforming/mixed-hybrid finite element scheme for degenerate parabolic problems. Numerische Mathematik 105.1 (2006): 73-131.

14. Eymard, R., Gallouet, T., and Herbin, R. : Finite volume methods. Handbook of numerical analysis 7 (2000): 713-1018.

15. Eymard, R., Herbin, R., and Michel, A. : Mathematical study of a petroleum-engineering scheme. ESAIM: Mathematical Modelling and Numerical Analysis 37.6 (2003): 937-972.

16. Hannukainen, A., Juntunen, M., and Stenberg, R. : Computations with finite element methods for the Brinkman problem. Computational Geosciences 15.1 (2011): 155-166.

17. Krotkiewski, M., Ligaarden, I. S., Lie, K. A., and Schmid, D. W. : On the importance of the stokes-brinkman equations for computing effective permeability in karst reservoirs. Communications in Computational Physics 10.05 (2011): 1315-1332.

18. Marusić-Paloka, E., Pazanin, I., and Marusić, S. : Comparison between Darcy and Brinkman laws in a fracture. Applied mathematics and computation 218.14 (2012): 7538-7545.

19. Misiats, O., and Lipnikov, K. : Second-order accurate monotone finite volume scheme for Richards equation. Journal of Computational Physics 239 (2013): 123-137.

20. Nasser El Dine, H., Saad, M., and Talhouk, R. : A convergent finite volume scheme of Darcy-Brinkman's velocities of twophase flows in porous media, accepted in proceeding ECMI 2016.

21. Saad, B., and Saad, M. A combined finite volum-nonconforming finite element scheme for compressible two phase flow in porous media. Numerische Mathematik 129.4 (2015): 691-722. 
22. Salinger, A. G., Aris, R., and Derby, J. J. : Finite element formulations for large-scale, coupled flows in adjacent porous and open fluid domains. International Journal for Numerical Methods in Fluids 18.12 (1994): 1185-1209.

23. Settari, A., and Aziz, K. : Treatment of nonlinear terms in the numerical solution of partial differential equations for multiphase flow in porous media. International Journal of Multiphase Flow 1.6 (1975): 817-844.

24. Vohralik, M. : On the discrete Poincare-Friedrichs inequalities for nonconforming approximations of the Sobolev space $H^{1}$. Numerical functional analysis and optimization 26.7-8 (2005): 925-952. 